\title{
Effect of Contrast and Adaptation on the Perception of the Direction and Speed of Drifting Gratings
}

\author{
ROLF MÜLLER, ${ }^{* \dagger}$ MARK W. GREENLEE* \\ Received 4 May 1993; in revised form 13 December 1993
}

\begin{abstract}
Three experiments were conducted to analyse the effect of contrast and adaptation state on the ability of human observers to discriminate the motion of drifting gratings. In the first experiment, subjects judged the direction of briefly presented gratings, which slowly drifted leftward or rightward. The test gratings were enveloped in space by a raised cosine function and in time by a Gaussian. The centre of the spatial envelope was either $\mathbf{2}$ deg left or right of the fixation point. An adaptive staircase procedure was used to find the velocities, at which the observer judged the motion direction in $75 \%$ of the presentations as leftwards or rightwards, respectively. In the second experiment, subjects judged the relative speed of two simultaneously presented gratings. Stimulus contrast was varied in both experiments from 0.01 to 0.32 . Discrimination threshold vs contrast functions were measured before and after adaptation to a high-contrast (0.4) grating drifting at rates between 2 and $32 \mathrm{~Hz}$. In a third experiment, subjects matched, before and after adaptation, the relative speed of a test stimulus, which had a constant contrast (0.04 or 0.08$)$ and a variable speed, to that of a reference stimulus having a variable contrast but a constant speed. The results indicate that, before adaptation, direction and speed diserimination thresholds are independent of test contrast, except when test contrast approaches the detection threshold level. Adaptation to a drifting grating increases the lower threshold of motion (LTM) and the speed discrimination threshold $(\Delta V / V)$ for low test contrasts. In addition, the point of subjective stationarity (PSS) shifts towards the adapted direction and this shift is more pronounced for low test contrasts. The perceived speed of a drifting grating increases with increasing contrast level. Adaptation to a drifting grating shifts the perceived speed vs log contrast function downwards and to the right (toward higher contrast levels) and this shift is greatest for adaptation frequencies between 8 and $16 \mathrm{~Hz}$. We further explored the effects of adaptation contrast $(0.04,0.4$ and 0.9$)$ and adaptation drift direction (iso- or contra-directional) on the perceived speed versus contrast function. The effect of adaptation is greatest for iso-directional drift and increases with increasing adaptation contrast. The results are discussed in terms of a contrast gain control model of adaptation.
\end{abstract}

Adaptation Motion perception Direction discrimination Speed discrimination

\section{INTRODUCTION}

Many aspects of vision improve with increasing stimulus contrast. Examples of this are performance on orientation and spatial frequency discrimination tasks. Discrimination performance increases for contrasts between the detection level and about 5 times this level, at which point it reaches its asymptotic value (Greenlee, 1992; Howard, 1989; Thomas, 1983). Contrast also plays an essential role in motion perception. For example, the perceived speed of a moving stimulus not only depends on its physical speed, but also on its contrast (Stone \& Thompson, 1992; Thompson, 1981). Perceived speed also depends on the stimulus spatial frequency (Boulton

*Neurologische Universitätsklinik, Abteilung für Neurophysiologie, Hansastr. 9, 79104 Freiburg, Germany.

†Carl-Ludwig-Institut für Physiologie, Universität Leipzig, Liebigstr. 27, 04103 Leipzig, Germany.
\& Baker, 1991; Diener, Wist, Dichgans \& Brandt, 1976; Ferrera \& Wilson, 1991; Smith \& Edgar, 1990), whether the stimulus contours are defined by luminance or colour differences (Cavanagh \& Favreau, 1985; Cavanagh, Tyler \& Favreau, 1984; Troscianko \& Fahle, 1988) and whether the stimuli are presented in central or peripheral vision (Hunzelmann \& Spillmann, 1984; Johnston \& Wright, 1986; Tynan \& Sekuler, 1982), as well as on the adaptation state of the observer.

Since the original studies of Pantle and Sekuler (1968) and Blakemore and Campbell (1969), we know that adaptation to high-contrast gratings increases the contrast detection threshold for test stimuli with similar spatial characteristics. More recently, Greenlee and Heitger (1988) reported that pattern adaptation can, under certain circumstances, improve contrast discrimination. Following adaptation to a high-contrast grating $(0.8)$, contrast discrimination thresholds were 
significantly lower for contrast levels above 0.5 . The improvement in contrast discrimination was also shown to be selective to the orientation of the test and adapting gratings. This enhancement in discrimination was taken as psychophysical evidence that contrast gain control can be a useful mechanism to insure a differentiated response at high-contrast levels. These results have recently been replicated and extended by Wilson and Humanski (1993), who proposed a neural network model of contrast gain control. There is ample evidence for contrast gain control in the response characteristics of cortical cells in the cat (Ohzawa, Sclar \& Freeman, 1985) and monkey (Albrecht, Farrar \& Hamilton, 1984; Sclar, Lennie \& DePriest, 1989). Albrecht et al. (1984) have shown that the hyperbolic ratio provides a good description of the contrast-response function of neurons in the visual cortex of the monkey. Adaptation appears to shift the semisaturation constant towards higher values, thereby unsaturating the adapted mechanisms.

The effects of adaptation on the relationship between contrast and the spatial frequency discrimination threshold for sine-wave gratings can also be accounted for by an increase in the semisaturation constant of the hyperbolic ratio (Greenlee \& Thomas, 1992). For spatial frequency discrimination, adaptation elevated discrimination thresholds at low contrasts, but had no effect on the asymptotic value of $\Delta f / f$ at high contrasts. Contrary to contrast discrimination, adaptation does not appear to have a direct benefit for spatial frequency discrimination. It remains to be determined whether adaptation can have a beneficial effect on other aspects of vision.

It has been shown that the perceived speed of drifting gratings depends on contrast (Stone \& Thompson. 1992; Thompson, 1982) and that adaptation to a moving grating can reduce the perceived speed of subsequently viewed stimuli (Thompson, 1981). In the present investigation, we determined the role of stimulus contrast and adaptation state on various aspects of the perception of drifting gratings. We demonstrate how adaptation to gratings drifting at different rates affects the relationship between contrast and the thresholds for discriminating the direction and speed of subsequently viewed drifting gratings. We further explore the effects of adaptation on the relationship between contrast and the perceived speed of drifting gratings. We propose that the effects of adaptation on motion perception can be described by changes in the underlying contrast transducer function. Adaptation acts to enlarge the range over which direction and speed discrimination thresholds depend on contrast. The perceived speed vs log contrast function is shifted downwards and to the right following adaptation to a high-contrast drifting grating. The results support a gain control model of adaptation, which is extended to explain the effects of contrast and adaptation on motion perception.

\section{METHOD}

\section{Stimuli}

Sine-wave luminance gratings of vertical orientation were produced on a high-resolution display (Joyce Electronics), having a white $(\mathbf{P 4})$ phosphor, a frame rate of $100 \mathrm{~Hz}$ and an average mean luminance of $200 \mathrm{~cd} / \mathrm{m}$. The linearity of the control voltage luminance characteristic of the display was calibrated using a spot photometer. The contrast of the grating stimuli was modulated in space by a raised cosine function and in time by a Gaussian envelope (Marcelja, 1980). The Gaussian had a time constant $\left(\sigma_{1}\right)$ of 11 frames, corresponding to $110 \mathrm{msec}$, i.e. $260 \mathrm{msec}$ above half-amplitude. In the space domain each grating had a total width of 2 deg. Contrast was defined by the Michelson equation and it varied from 0.01 to 0.32 . In all experiments, the spatial frequency of the test gratings was $1.6 \mathrm{c}$ deg. The test gratings were presented 2 deg on either side of the central fixation point. In Expts I and II, the adapting grating subtended $10 \mathrm{deg}$ in width and was centred in the middle of the display, whereas in Expt III it was positioned on the right half of the screen. Its spatial frequency was also constant at $1.6 \mathrm{c} / \mathrm{deg}$. In Expt I, the drift frequency of the adapting grating was either 2 or $8 \mathrm{~Hz}$ and in Expt II. it varied from 2 to $32 \mathrm{~Hz}$. In both experiments adaptation direction was rightwards and adaptation contrast was 0.4 . In Expt III, the adapting grating drifted at rates between 2 and $32 \mathrm{~Hz}$ to the right or to the left. Adaptation contrast was either $0.04,0.4$ or 0.9 . In all experiments, a constant interval of $2 \mathrm{sec}$ was interposed between the end of the readaptation period and the beginning of the test period, during which time the display was blank. Figure 1 illustrates the three experimental conditions.

\section{Procedure}

The observers viewed the display binocularly at a distance of $0.84 \mathrm{~m}$. During the experiments, they were asked to fixate a point positioned in the centre of the display. The latter was surrounded by a semi-circular, back-illuminated Plexiglas screen with a mean luminance of $100 \mathrm{~cd} / \mathrm{m}^{2}$. Viewing distance and head orientation was held constant by having observers place their chin on a chin-forehead rest.

Experiment I: direction discrimination. Figure I(a) schematically illustrates the stimulus conditions used in this experiment. In a typical run, four different contrast values were presented in random order, each contrast 20 times. On each trial, one stimulus was randomly presented either $2 \mathrm{deg}$ left or right of the fixation point. The stimulus drifted either to the left or to the right and the direction of this drift was random from trial to trial. Approximately $300 \mathrm{msec}$ before the presentation of each stimulus, a computer-generated tone was given to announce the beginning of the next trial. The observer judged in which direction the grating drifted. The drift velocity was controlled using an adaptive, maximum likelihood algorithm (Lieberman \& Pentland, 1982). The velocity of a stimulus perceived in $75 \%$ of the presentations as leftwards drifting was defined as the leftward direction threshold and the velocity of a stimulus perceived in $75 \%$ of the presentations as rightwards drifting was defined as the rightward direction threshold. 


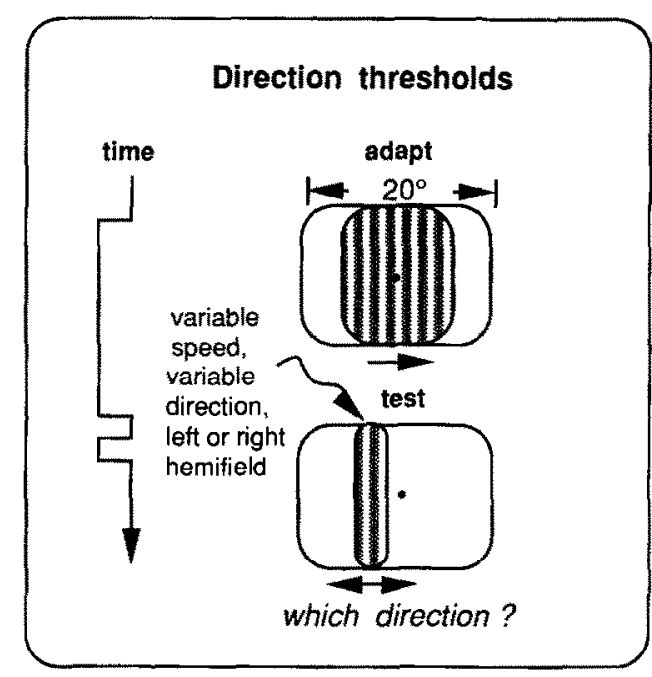

b

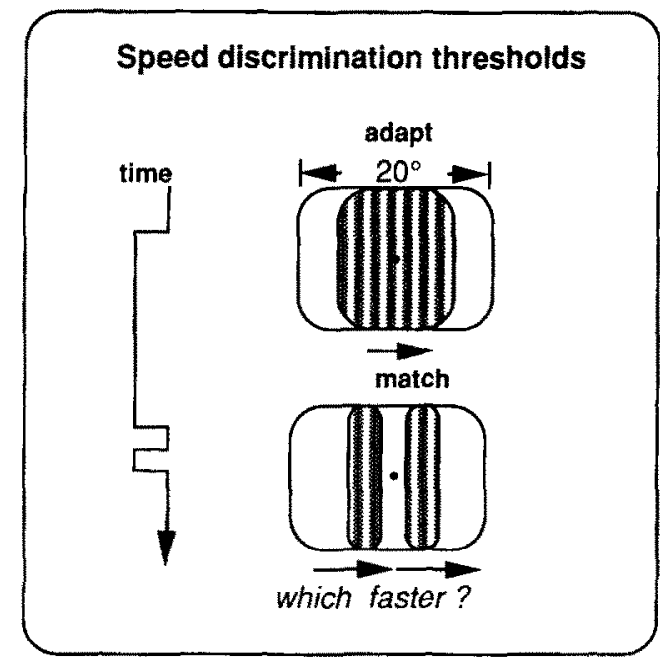

C

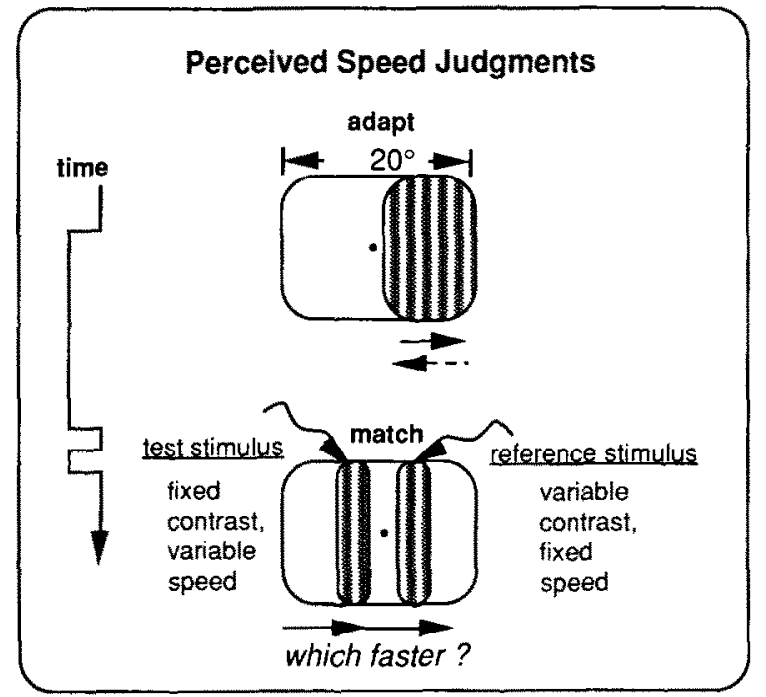

FIGURE 1. Schematic illustration of the stimulus conditions used in the experiments. (a) The conditions used in the direction threshold measurements before and after adaptation to a rightward drifting grating. On each trial, a test grating was presented randomly left or right of fixation. Its direction varied randomly either to the left or right. The observer judged in which direction the grating moved. (b) The stimulus conditions used to determine the speed discrimination thresholds before and after adaptation to a rightward drifting grating. On each trial, two gratings were presented left and right of fixation during the match period. One had the reference speed and one had the test speed. The observer judged which grating moved faster. (c) The experimental paradigm used to estimate the perceived speed of drifting gratings before and after adaptation to a rightward or leftward drifting grating, positioned 1 deg off-centred in the right visual ficld.

Direction thresholds were determined before and after adaptation. The subjects viewed the adapting grating for $100 \mathrm{sec}$. The first trial began $2 \mathrm{sec}$ after the adaptation grating was turned off. Each trial was followed by a $10 \mathrm{sec}$ readaptation period, in which the same adaptation grating was presented again. Two runs were conducted for each condition.

Experiment II: speed discrimination. Figure 1(b) schematically illustrates the stimulus conditions used in this experiment. Speed discrimination was measured for two spatially truncated sine-wave gratings, presented simultaneously 2 deg on either side of a fixation point. In a typical run, four different contrast values were presented in random order. Both gratings always had the same contrast. One of the gratings had the reference speed of
$1.25 \mathrm{deg} / \mathrm{sec}$ (or $2 \mathrm{~Hz}$ ) and the other had the test speed, which was incremented by a certain amount $(\Delta V)$. The position of the test and reference grating was randomly determined on each trial. The subject responded which grating appeared to move faster. Individual maximumlikelihood estimates of the threshold $\Delta V$ value, where threshold performance was defined as $75 \%$ correct, were made for each of the reference contrast levels using the Best-PEST procedure. Speed discrimination thresholds were measured before and after adaptation. Adaptation and match drift directions were always to the right. The initial adaptation period was $100 \mathrm{sec}$. As in Expt I, each match period was followed by a readaptation period of $10 \mathrm{sec}$ duration. Three runs were performed for each condition. 
Experiment III: perceived speed judgements. Figure I(c) illusirates the stimulus conditions used. The perceived speed of the reference grating drifting at a rate of $2 \mathrm{~Hz}$ was determined using a modified two-alternative forced-choice paradigm. The contrast of the reference grating was varied from 0.01 to 0.32 in separate staircases. Its speed was compared to that of the test grating, which had a variable speed but a fixed contrast of 0.04 or 0.08 , depending on the conditions investigated. Approximately $300 \mathrm{msec}$ before the presentation of each stimulus pair, a computer-generated tone was given to announce the beginning of the next trial. The subjects were asked to judge which of the two gratings appeared to move faster. In a typical run, four or five different contrast values of the reference grating were presented in random order, each stimulus 20 times. Individual maximum-likelihood estimates of the speed of the test grating which yielded $50 \%$ "faster" judgements were determined [Best-PEST (Lieberman \& Pentland, 1982)]. The speed judgements were made before and after adaptation. The initial adaptation period was $100 \mathrm{sec}$ and each match period was followed by a readaptation period of $10 \mathrm{sec}$ duration. Three runs were performed for each condition.

\section{Observers}

The authors served as the two main observers. A third subject, HJL, was run in Expt I and the first part of Expt II. A fourth subject, EMM, was run in Expt III. Observers HJL and EMM were uninformed as to the aims of the investigation. The two main observers and EMM wore their refractive corrections during the experiments. HJL is emmetropic. Viewing was binocular and conducted with natural pupils. All subjects were well- practised psychophysical observers, so that they were able to fixate a point in the centre of the screen despite the presentation of a leftward or rightward drifting grating. During adaptation and test/match periods, eye movements were restricted, for the most part. to physiological fixation nystagmus.

\section{RESULTS}

\section{Experiment I: direction discrimination}

The results of the first experiment are shown in Fig. 2. Prior to adaptation, direction thresholds are more or less independent of test contrast. For subject MWG [Fig. 2(a)], the values range from 0.1 to $0.35 \mathrm{~Hz}$ (corresponding to $0.06-0.22 \mathrm{deg} / \mathrm{sec}$ ) for perceived rightward drift. These findings are in close agreement with published results for similar stimulus conditions (Johnston \& Wright, 1985; Wright \& Gurney, 1992). The rightward direction thresholds are elevated following adaptation to a grating drifting at $2 \mathrm{~Hz}$ and this elevation is most pronounced for low test contrasts. Adaptation to an $8 \mathrm{~Hz}$ drift frequency has an even more pronounced effect on the rightward direction threshold. In a similar manner, the leftward direction thresholds are shifted in the rightwards direction after $2 \mathrm{~Hz}$ rightward adaptation and by an even greater amount after $8 \mathrm{~Hz}$ rightward adaptation. At low test contrasts, following adaptation the test stimuli were more difficult to detect and determination of direction thresholds becomes less reliable. The leftward direction thresholds cross the zero line and take on positive values at intermediate contrast levels.

The results of the second and third observers are shown in Fig. 2(b,c), and confirm these findings.

a

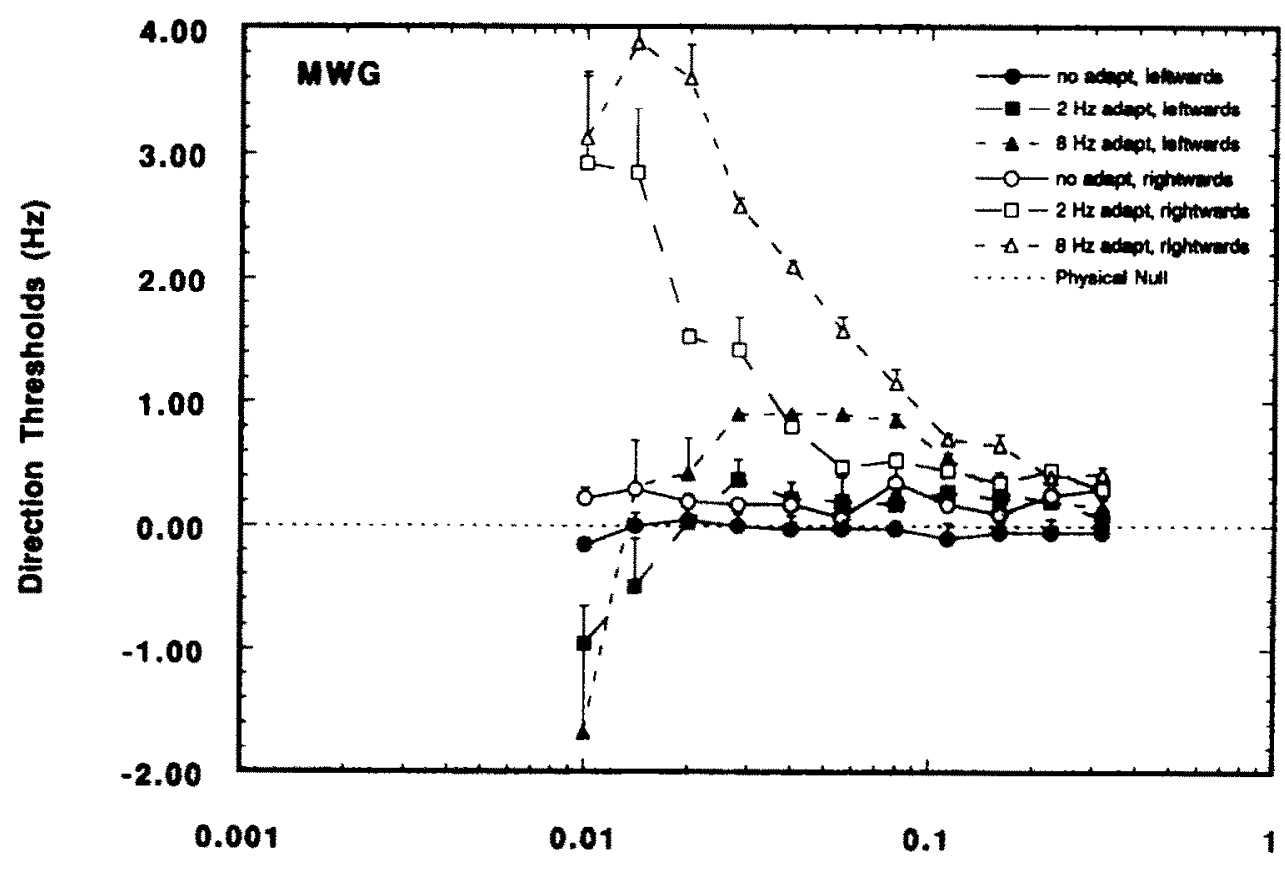

Test Comrast

FIGURE 2(a). Caption on facing page. 
b

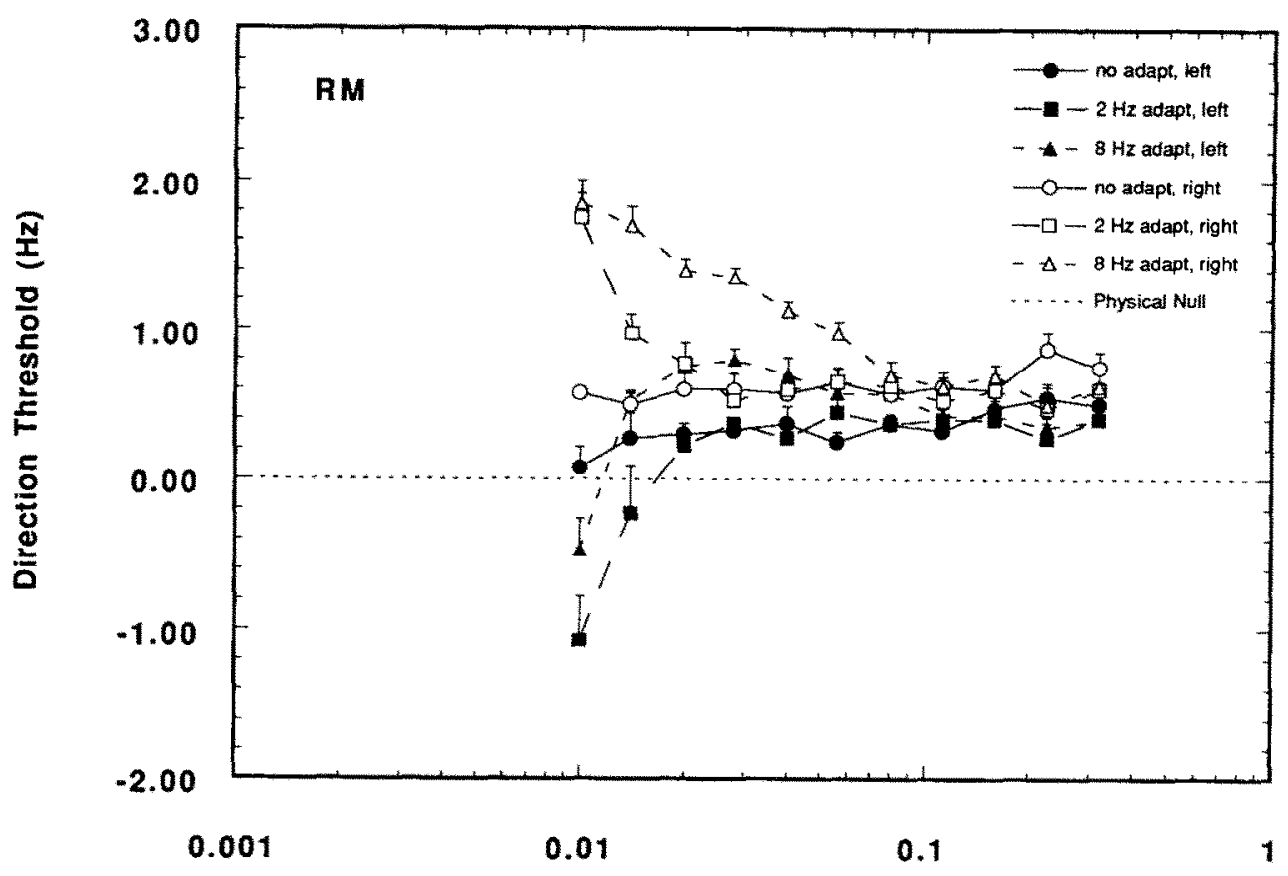

Test Contrast

C

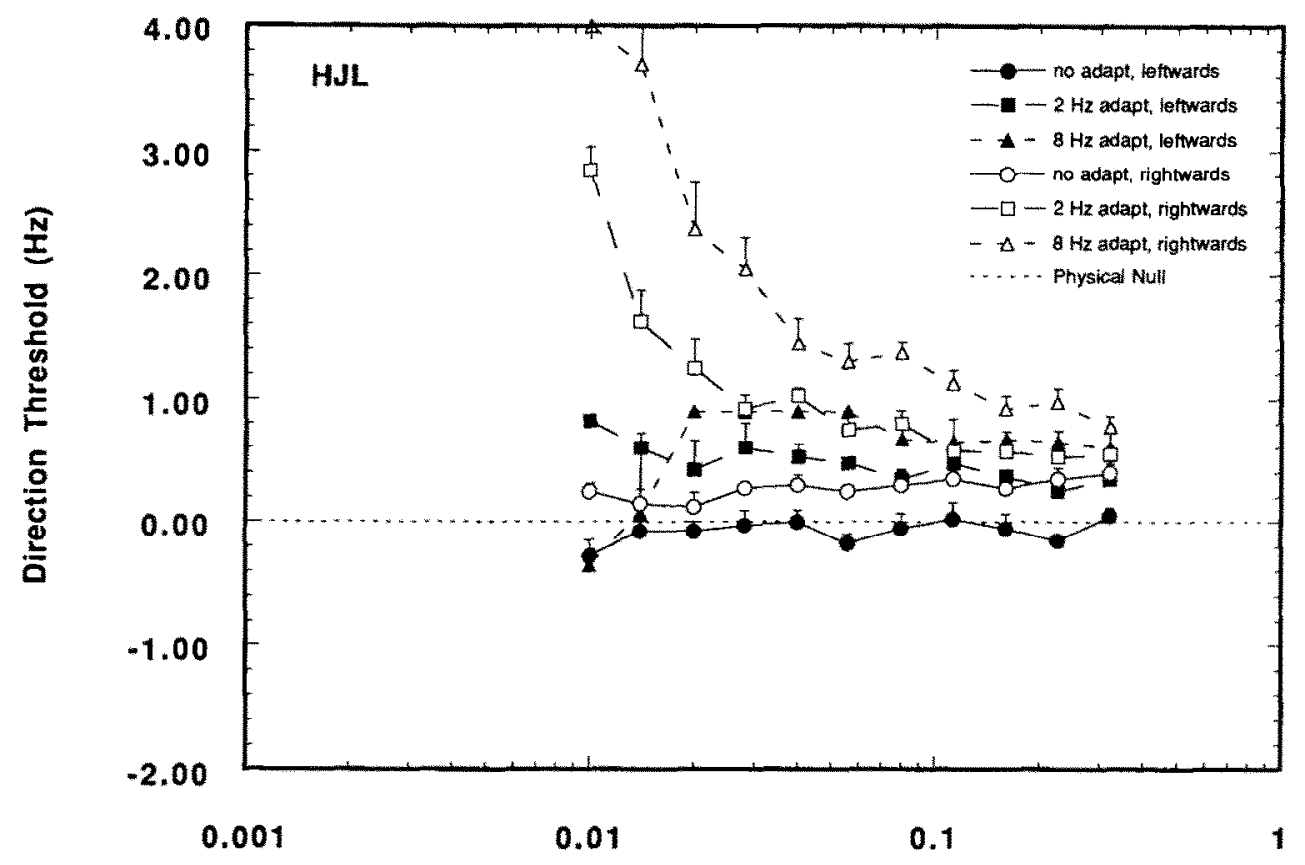

Test Contrast

FIGURE 2. Direction thresholds are plotted as a function of the test contrast on semi-logarithmic coordinates. The mean values of two runs, averaged over the left and right visual hemifield $(n=4)+1 \mathrm{SE}$, are shown. Positive values designate rightward motion and negative values represent leftward motion. Circles present the results determined before adaptation, squares show the findings measured after $2 \mathrm{~Hz}$ adaptation rightward and triangles give the results for measurements made after $8 \mathrm{~Hz}$ adaptation rightward. Open symbols present the findings for the rightward direction thresholds and solid symbols give the results for the leftward direction thresholds. The dashed horizontal line depicts physical stationarity. (a) The findings for observer MWG, (b) for RM and (c) for HJL. An analysis of variance for repeated measures (Super ANOVA, version 1.1, Abacus Concepts, U.S.A.) was conducted to test the statistical significance of the effects of adaptation state, test contrast, drift direction and position (left or right visual hemifield) on direction thresholds. This analysis revealed significant main effects of Adaptation State [i.e. no adaptation, 2 and $8 \mathrm{~Hz}$ adaptation; $F(2,6)=10.75, P=0.01$ ], Test Contrast $[F(10,60)=6.4$, $P=0.0001]$ and Drift Direction $[F(1,6)=45.3, P=0.0005]$. The effect of the visual hemifield (i.e. stimulus presented left or right of fixation) was not significant. There were also significant interactions between the main effects Adaptation State and Test Contrast $[F(20,60)=3.7, P=0.0001]$, Adaptation State and Drift Direction $[F(2,6)=4.9, P=0.05]$, Test Contrast and Drift Direction $[F(10,60)=23.0, P=0.0001]$, as well as between all three factors $[F(20,60)=5.8, P=0.0001]$. 
Observer RM's unadapted thresholds are somewhat shifted towards rightward drift direction, a trend which is also evident, though to a lesser extent, in the two other subjects. In addition the effects of adaptation on RM`s threshold data are less pronounced than those of observers MWG and HJL (note different ordinate scales). Otherwise the results of the three subjects are similar.

The results of an analysis of variance are summarized in the legend of Fig. 2. The effects of test contrast and adaptation state are highly significant. The significant interaction between adaptation state and test contrast implies that the shape of the direction thresholds vs contrast functions differs after adaptation. The interaction between adaptation state and drift direction implies that the adaptation effect had a significant direction-specific component. As is evident in Fig. 2, the effects of iso-directional adaptation are more pronounced than those of contra-directional adaptation. The test contrast $x$ drift direction interaction, as well as the three-way interaction suggests that the shape of these functions also depends on the drift direction, especially after adaptation. Again, these trends are evident in the results presented in Fig. 2.

From the results in Fig. 2 we can calculate the lower threshold of motion (LTM) as the difference between the thresholds for perceiving rightward and leftward motion divided by two, in analogy to Johnston and Wright (1985). Figure 3 plots LTM as a function of test contrast. The different symbols present the findings for the three adaptation conditions (see inset). The curves present the best fitting hyperbolic ratios for the reciprocal value of LTM (i.e. the absolute motion sensitivity):

$$
\frac{1}{\mathrm{LTM}}=K\left[\begin{array}{c}
C^{\prime \prime} \\
\left(C^{n}+\bar{C}_{n s}^{n}\right)
\end{array}\right]
$$

where $K=1 / \mathrm{LTM}_{\min }$. $\mathrm{LTM}_{\min }$ is the asymptotic value of LTM at high contrasts, $C_{0}$; is the semisaturation constant of the contrast transducer function and the exponent $n$ determines the steepness of the function when $1 /$ LTM is plotted against $\log C$ (Greenlee \& Thomas, 1992). As can be seen in Fig. 3, the hyperbolic ratio provides a convenient description of the relationship between $1 /$ LTM and log contrast. The correlation coefficients describing the goodness of fit were 0.44, 0.7 and 0.56 for the unadapted condition, $0.98,0.99$ and 0.98 for the adapted conditions, for subject MWG, RM and HJL, respectively. As is evident from the results, adaptation increases the contrast dependency of LTM and the curves describing these functions shift towards higher contrast levels.

The results shown in Fig. 2 also allow us to analyse the effects of adaptation on the point of subjective stationarity (PSS). Stimuli at that point are judged in $50 \%$ of the presentations as rightward and $50 \%$ as leftward. We calculate PSS as the sign-conserving mean of the leftwards and rightwards direction thresholds and as such it represents a statistical estimate of the PSS. The results from the three observers are shown in Fig. 4(a-c). Following adaptation to a rightward drifting grating PSS is shifted towards higher velocities of rightward motion. The magnitude of this shift depends on the test contrast: the effects of adaptation are most pronounced for low test contrasts. All subjects show a more pronounced adaptation effect for the higher adaptation frequency of $8 \mathrm{~Hz}$. This shift in PSS corresponds to the well-known aftereffect of motion adaptation, in which

a

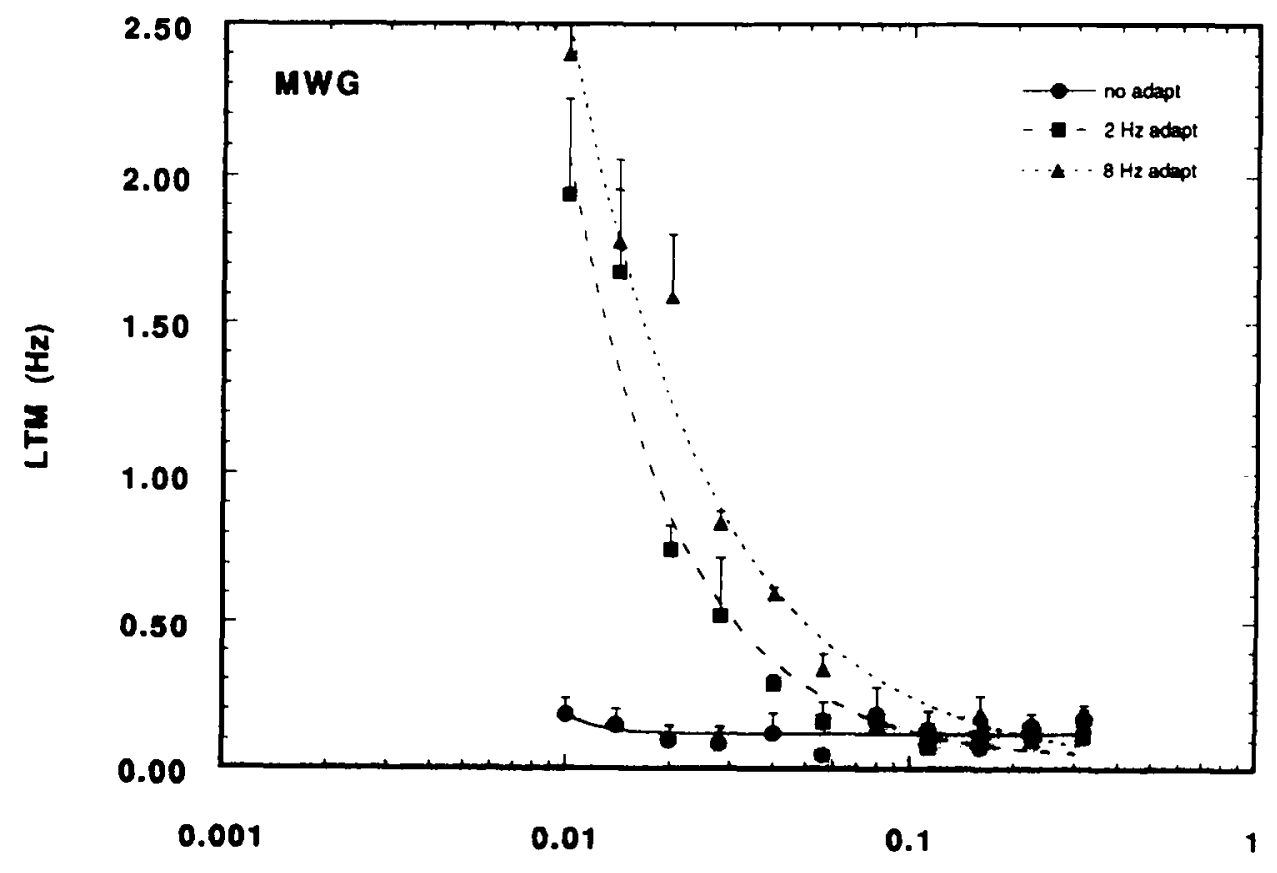

Test Contrast

FIGURE 3(a). Caption on facing page. 
b

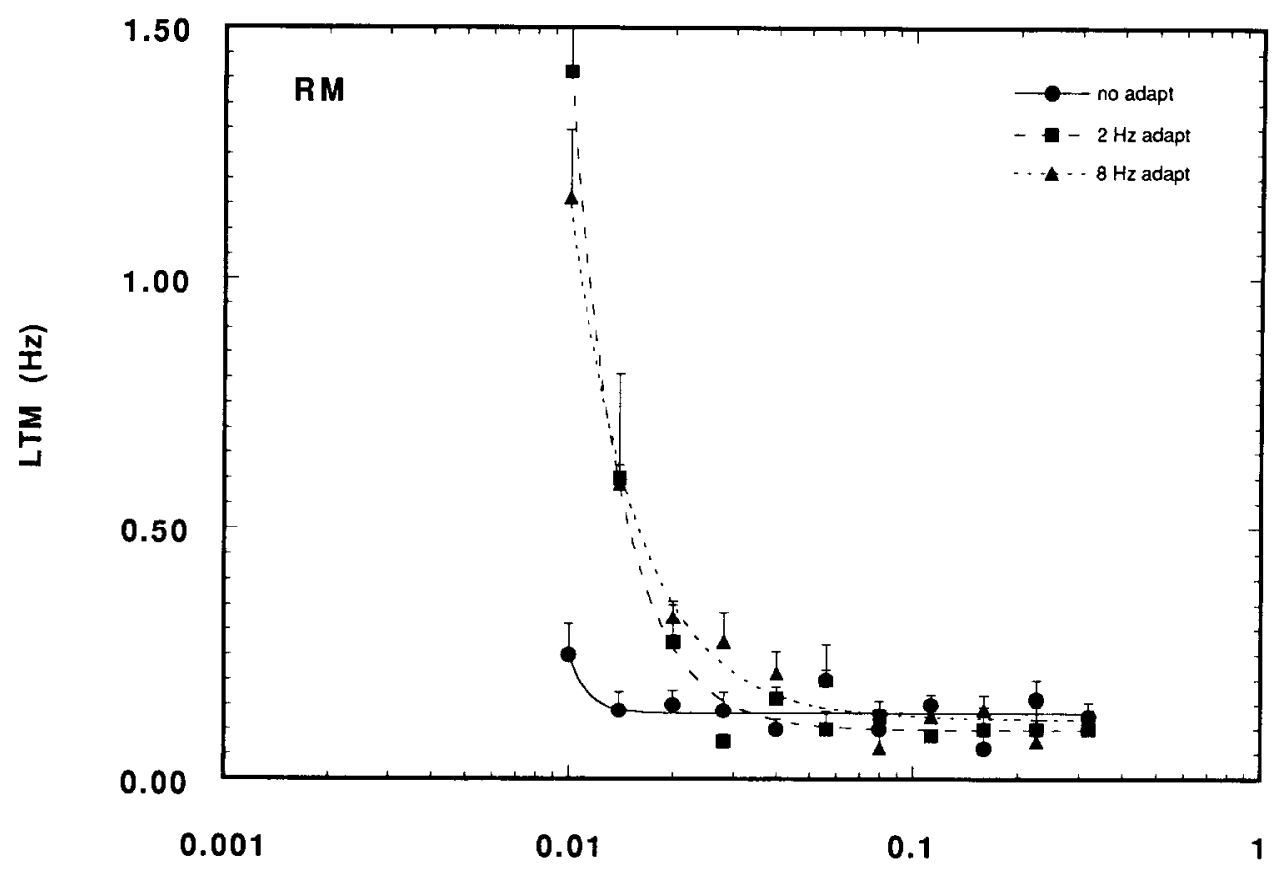

Test Contrast

C

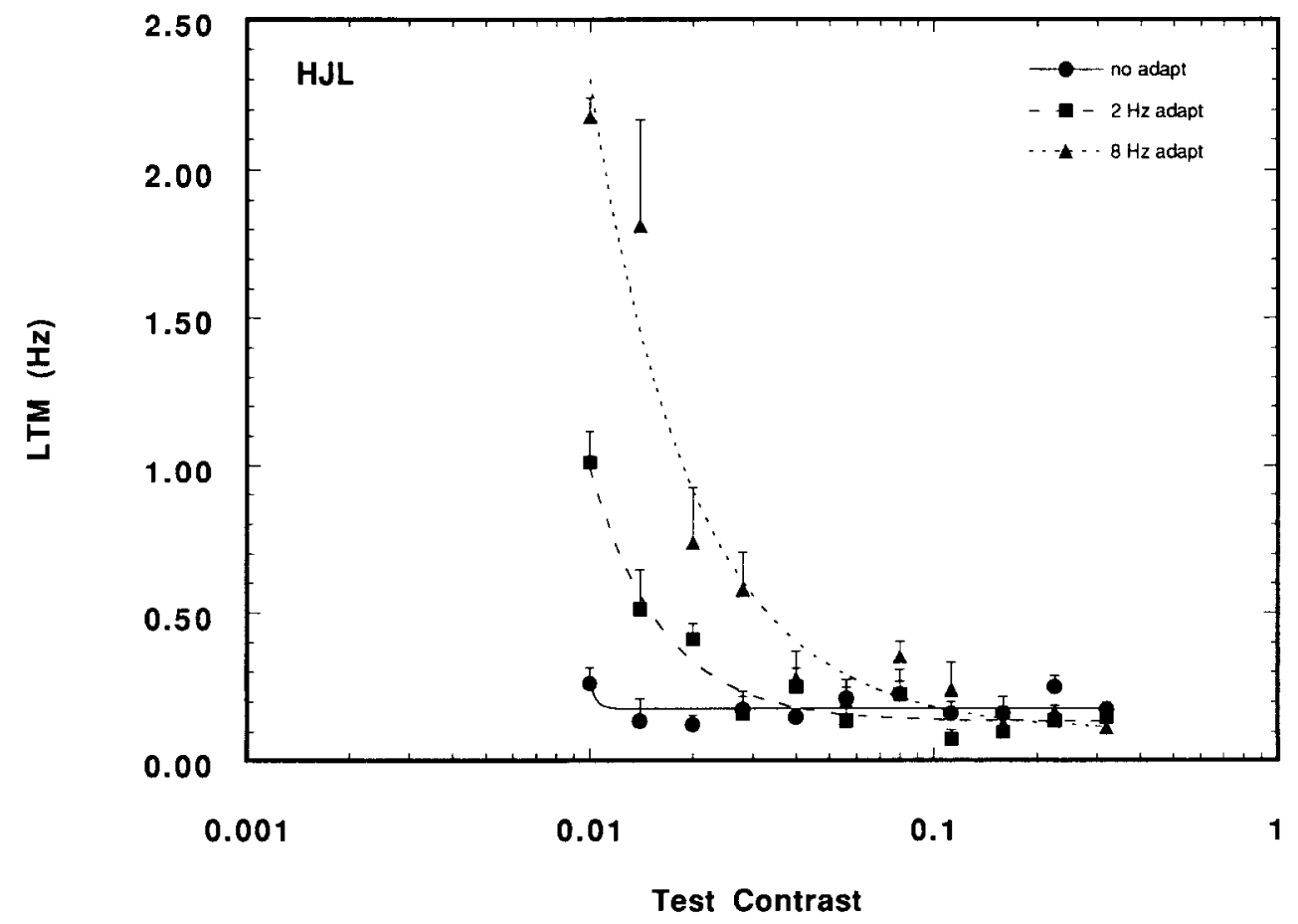

FIGURE 3. The LTM, defined as the difference between rightward and leftward direction thresholds divided by 2, is plotted as a function of test contrast on semi-logarithmic coordinates. Circles present the results determined prior to adaptation, squares show the findings measured after $2 \mathrm{~Hz}$ adaptation and triangles give the results for measurements made after $8 \mathrm{~Hz}$ adaptation. The symbols indicate the mean values of two runs, for each of the two hemifields $(n=4)$ and error bars show + 1SE. The curves are the best fitting hyperbolic ratios of 1/LTM. (a-c) The findings for the observers MWG, RM and HJL, respectively. 
stationary gratings appear to drift in a direction opposite that of the adapting grating (Barlow \& Hill, 1963; Sekuler \& Pantle, 1967).

\section{Experiment II: speed discrimination}

The results of the second experiment, in which we explored the effects of adaptation on the speed discrimi- nation threshold (i.e. the Weber fraction $\Delta V / V$ ) are shown in Fig. 5. Figure 5(a) presents the results from observer MWG and Fig. 5(b) gives the results for observer RM. Only uncomplete data for the unadapted and $2 \mathrm{~Hz}$ adaptation condition were collected in subject HJL, and, though similar to those of the two main observers, are not shown in Fig. 5. $\Delta V / V$ decreases with

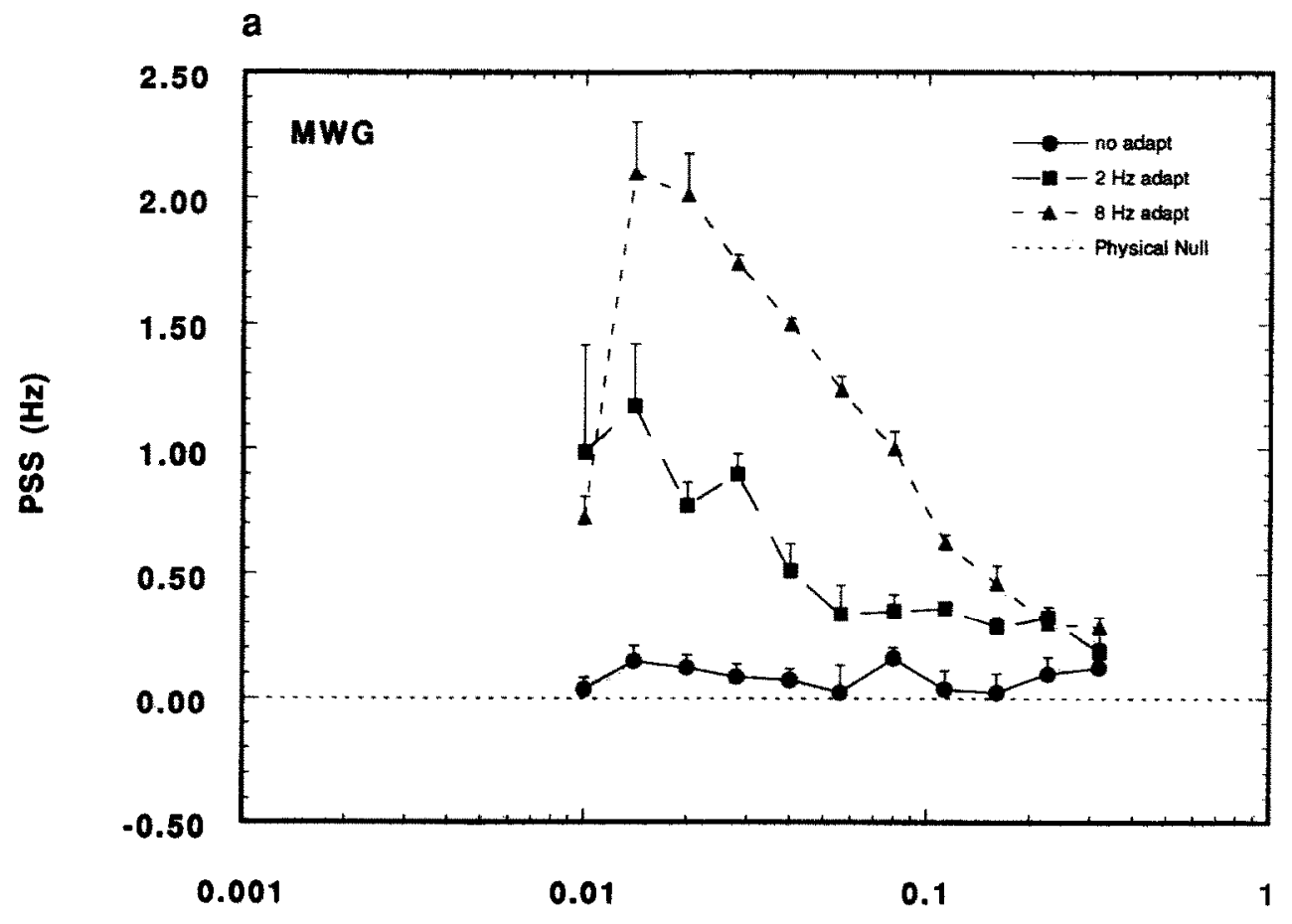

Test Contrast

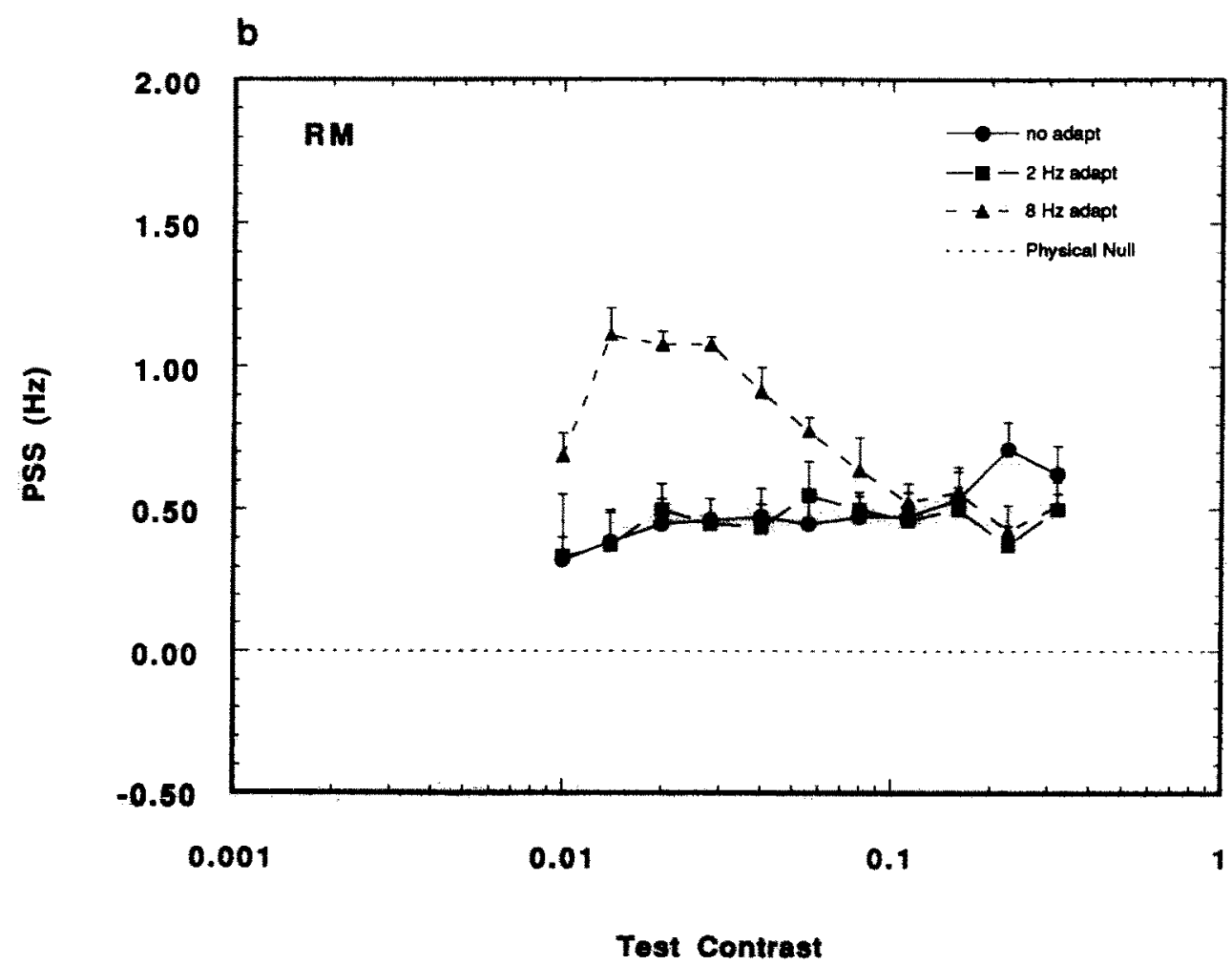

FIGURE 4(a,b). Caption on facing page. 


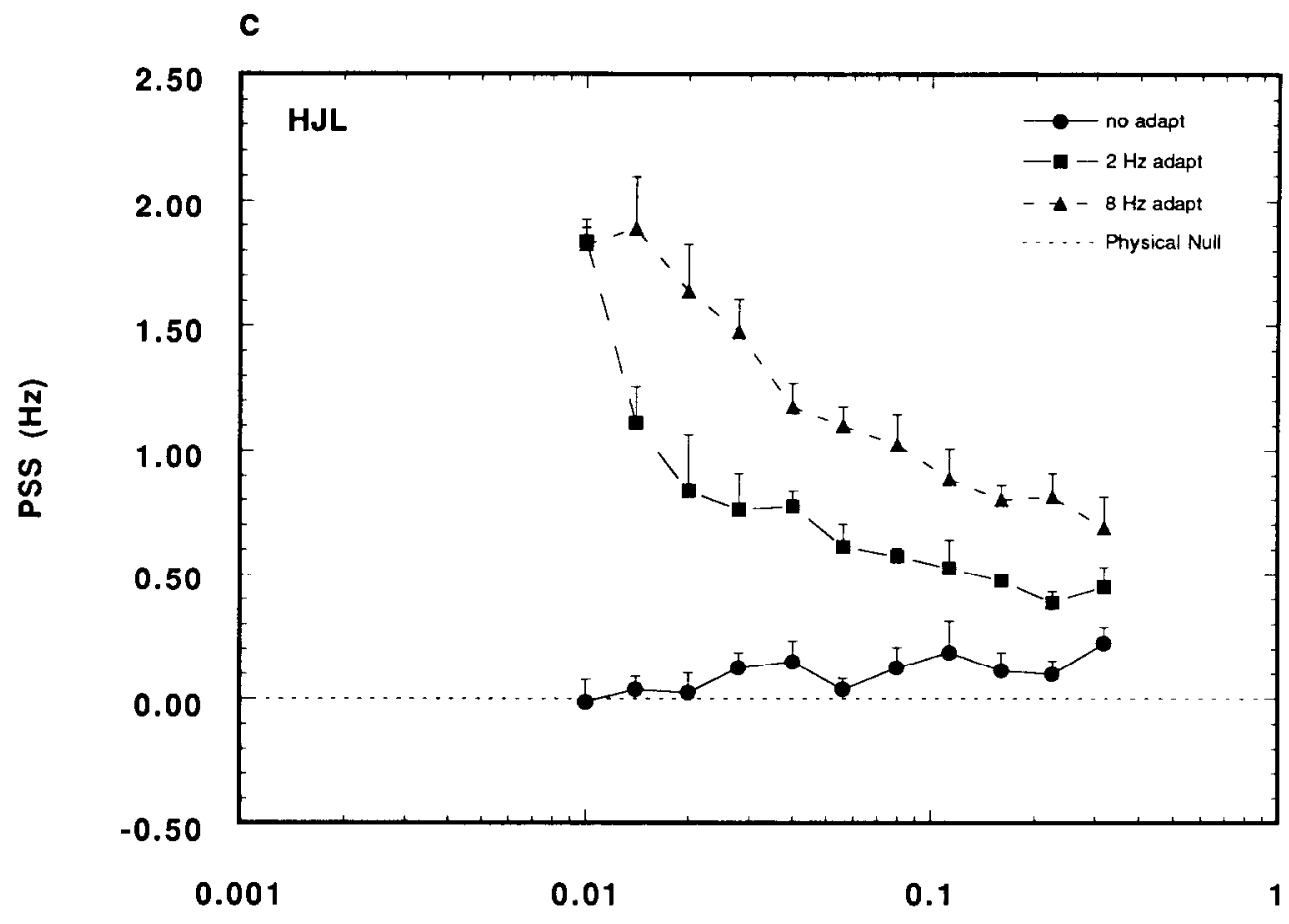

Test Contrast

FIGURE 4. The PSS, defined as the mean of the direction thresholds for leftward and rightward motion, is shown as a function of the test contrast on semi-logarithmic coordinates. Circles present the results determined before adaptation, squares show the findings measured after $2 \mathrm{~Hz}$ adaptation rightward and triangles give the results for measurements made after $8 \mathrm{~Hz}$ adaptation rightward. The symbols indicate the mean values of two runs, for each of the two hemifields $(n=4)$ and crror bars show + 1SE. (a-c) The findings for observers MWG, RM and HJL, respectively.

increasing contrast level and this contrast dependency becomes more pronounced after adaptation. Adaptation shifts the discrimination function to the right. The curves are the best fitting hyperbolic ratios of the form:

$$
\frac{V}{\Delta V}=\frac{V}{\Delta V_{\min }}\left[\frac{C^{n}}{\left(C^{n}+C_{0.5)}^{n}\right.}\right]
$$

where $\Delta V_{\min }$ is the asymptotic value of $\Delta V$ for high reference contrasts, $\mathrm{C}_{0.5}$ is the scmisaturation constant, i.e. the contrast value at which $V / \Delta V$ is at half its maximal value and the exponent $\mathrm{n}$ is the steepness parameter.

The correlation coefficient describing the goodness of fit was 0.66 (observer MWG) and 0.85 (observer RM) for the unadapted thresholds and 0.900 .99 for the adapted thresholds. An analysis of variance for repeated measures revealed highly significant effects of reference contrast $[F(10,40)=7.3, P=0.0001]$ and adaptation state $[F(5,20)=3.8, \quad P=0.014]$ on discrimination thresholds. The interaction between these terms was also highly significant $[F(50,200)=2.8, P=0.0001]$, indicating that the shapes of the unadapted and adapted functions significantly differ from each other.

The results presented in Fig. 5 clearly indicate that, following adaptation to a rightward drifting grating of 0.4 contrast, speed discrimination thresholds are elevated for low contrast gratings. The amount of this threshold elevation can be expressed by the ratio,

$$
R_{\Delta V}=\frac{\Delta V_{\text {adapted }}}{\Delta V_{\text {unadapted }}}
$$

and this ratio is plotted in Fig. 6 as a function of the adaptation drift rate for three different reference contrast levels (see inset). For high reference contrast levels, adaptation has no effect on speed discrimination thresholds $\left(R_{\Delta V} \approx 1.0\right)$. The results for low reference contrast levels indicate that the adaptation effect is dependent on the temporal rate of the adapting grating: thresholds are most greatly elevated by adaptation ranging between 8 and $16 \mathrm{~Hz}$. As will be shown below, it is just at these adapting frequencies that the perceived speed of a drifting grating is maximally reduced.

\section{Experiment III: effect of iso-directional adaptation on speed judgements}

The results of Expt III, in which we explore the effect of adaptation on the perceived speed of drifting gratings, are shown in Fig. 7 for two observers. The perceived speed of the reference grating is represented by $V^{\prime \prime}$, where

$$
V^{\prime}=\frac{V_{\text {test }}}{V_{\text {reference }}}
$$

is plotted as a function of the reference contrast on semi-logarithmic coordinates. A $V^{\prime}$ of 1 means that the speed of the reference grating was perceived veridically. A value $<1.0$ means that the speed of the test grating had to be reduced in order to match that of the reference 

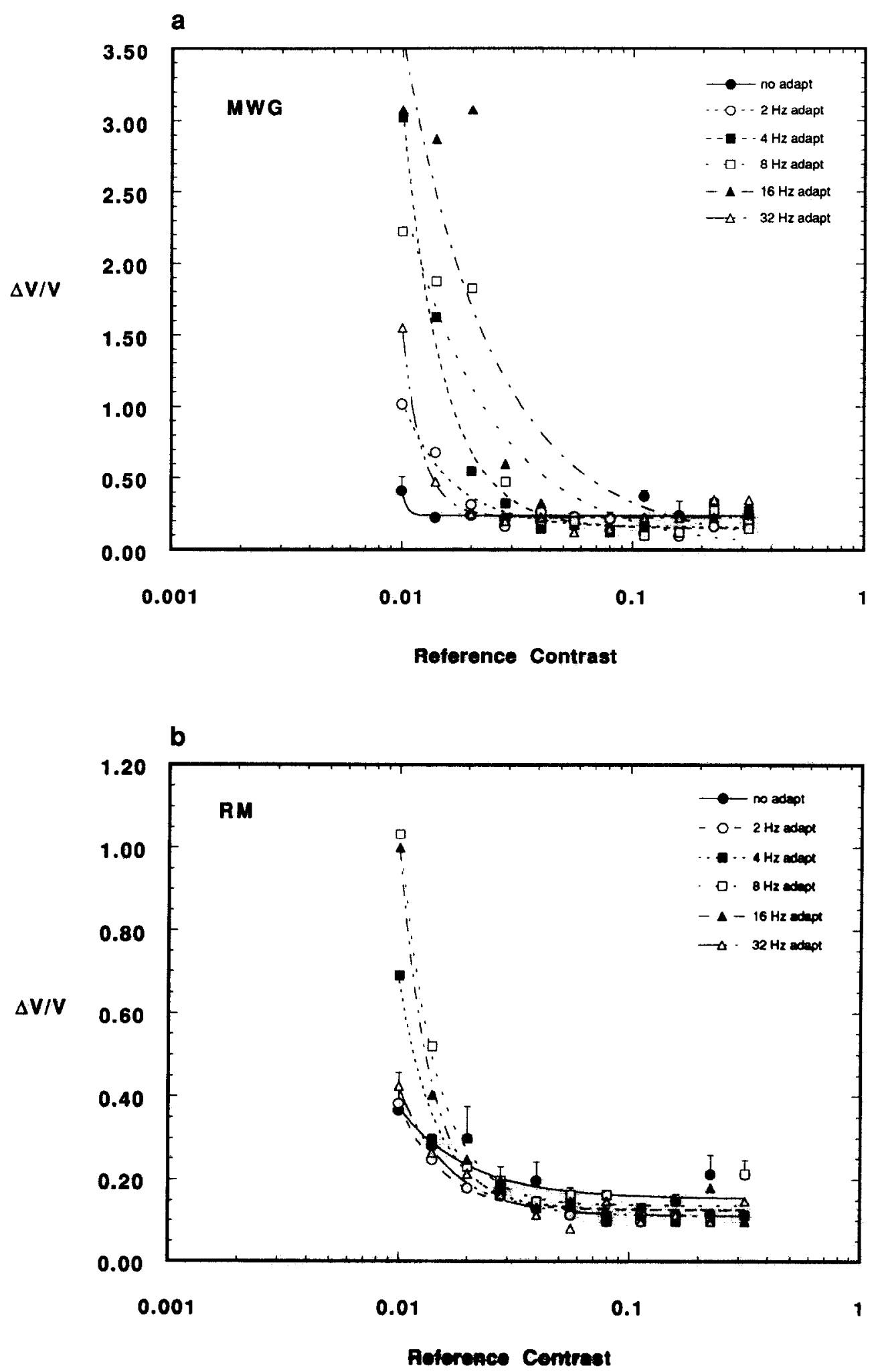

FIGURE 5. Speed discrimination thresholds (i.e. the Weber fractions $\Delta V / V$ ) are shown as a function of reference contrast on semi-logarithmic coordinates. Solid circles present the results determined before adaptation and the other symbols show the results after adaptation to rightward drifting gratings of various tomporal frequency $(2-32 \mathrm{~Hz}$; see inset). The symbols present the means of three runs and error bars show + ISE. For the sake of clarity, error bars are given only for the unadapted values, which are similar in size to the adapted values. The curves show the best fitting hyperbolic ratios for $V / \Delta V$. (a) The results for observer MWG and (b) those of observer RM. 
a

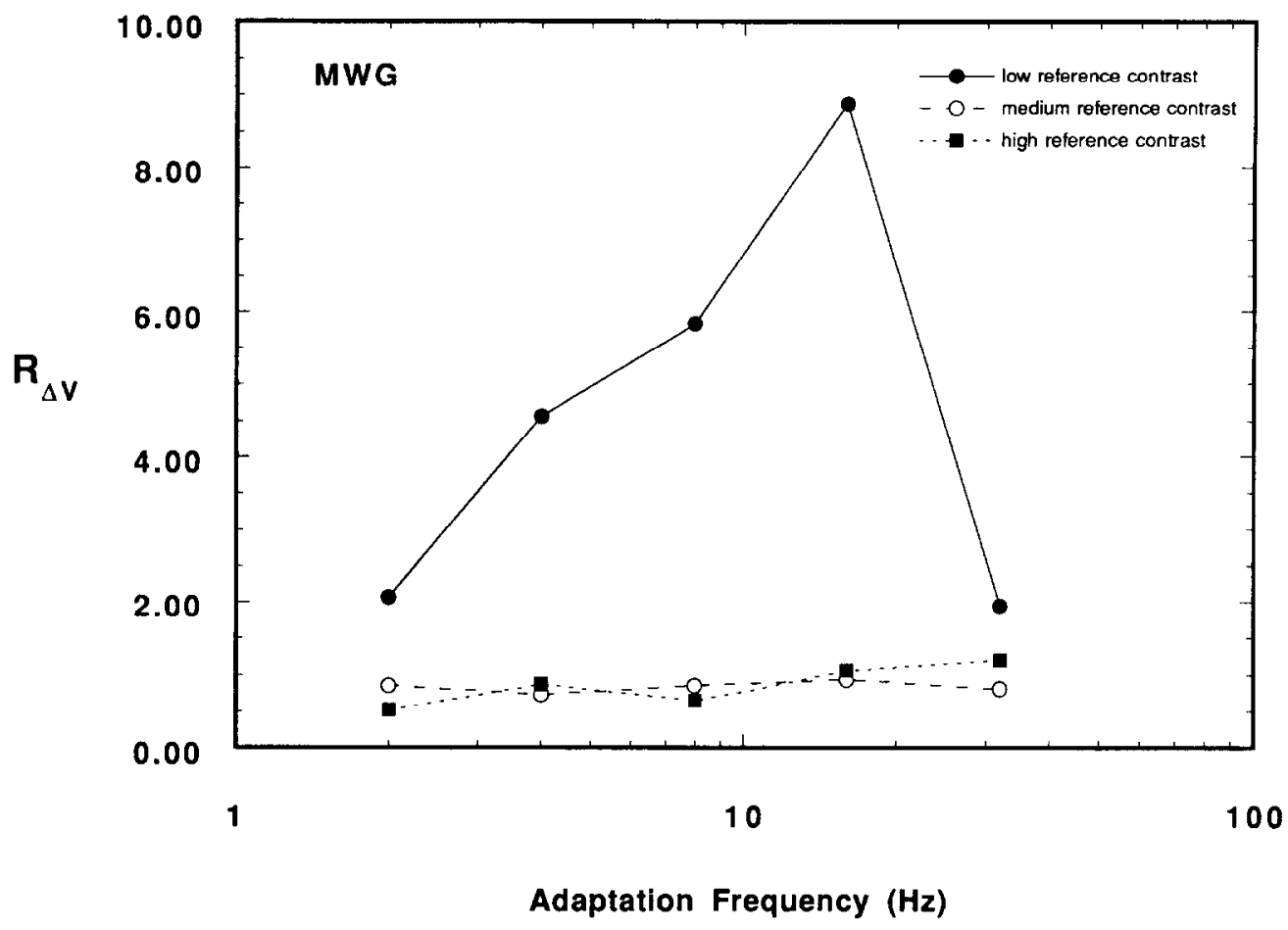

b

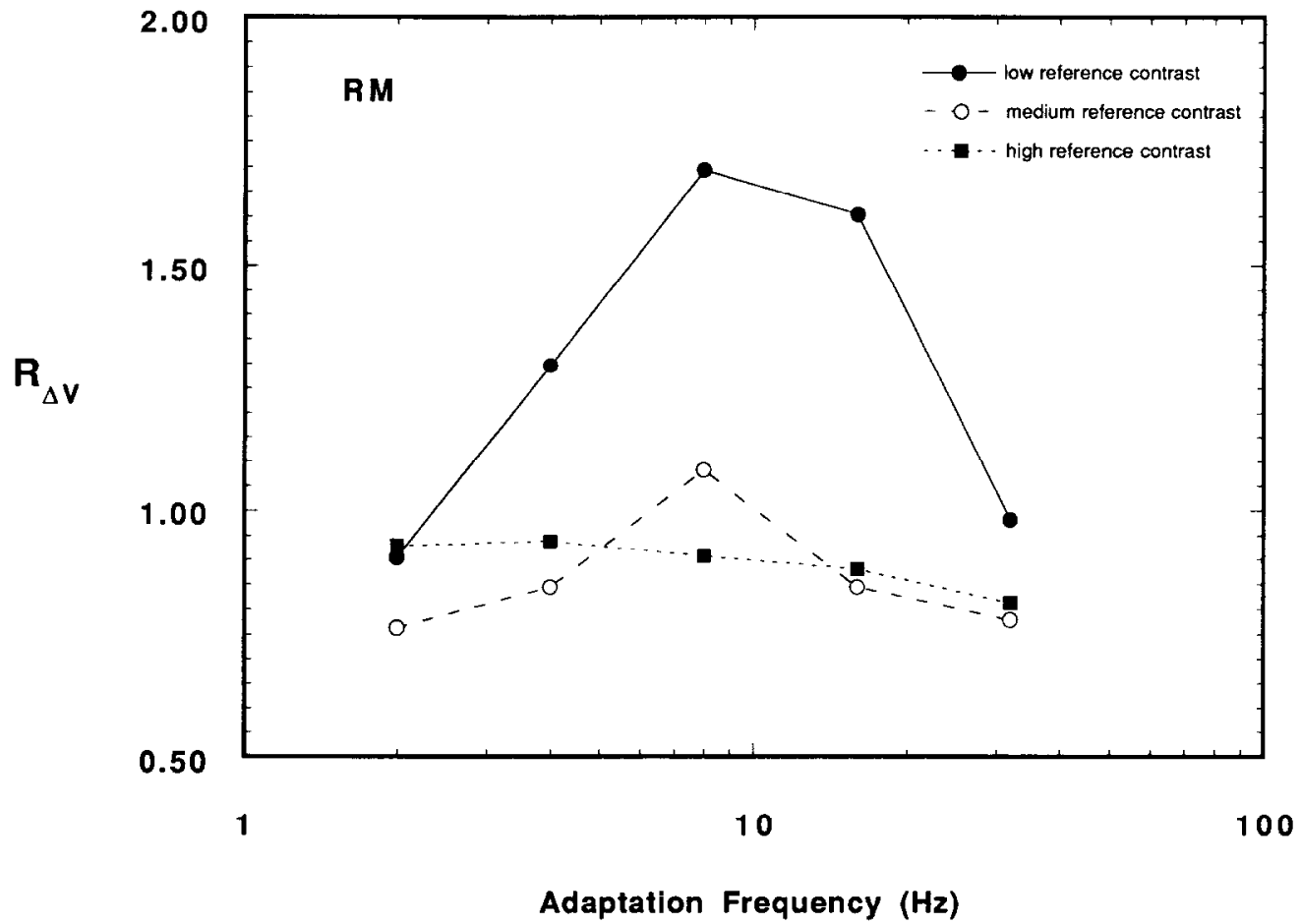

FIGURE 6. The relative adaptation effect $R_{\mathrm{A} V}$, as defined in equation (3), is plotted as a function of the adapting temporal frequency for observer MWG (a) and RM (b). The solid circles show the mcan values for the reference contrasts $0.01,0.014$, 0.02 and 0.028 (low reference contrasts), the open circles present the mean values for $0.04,0.056$ and 0.08 (medium reference contrasts) and solid squares give the mean values for $0.113,0.16,0.226$ and 0.32 (high reference contrasts). 
a

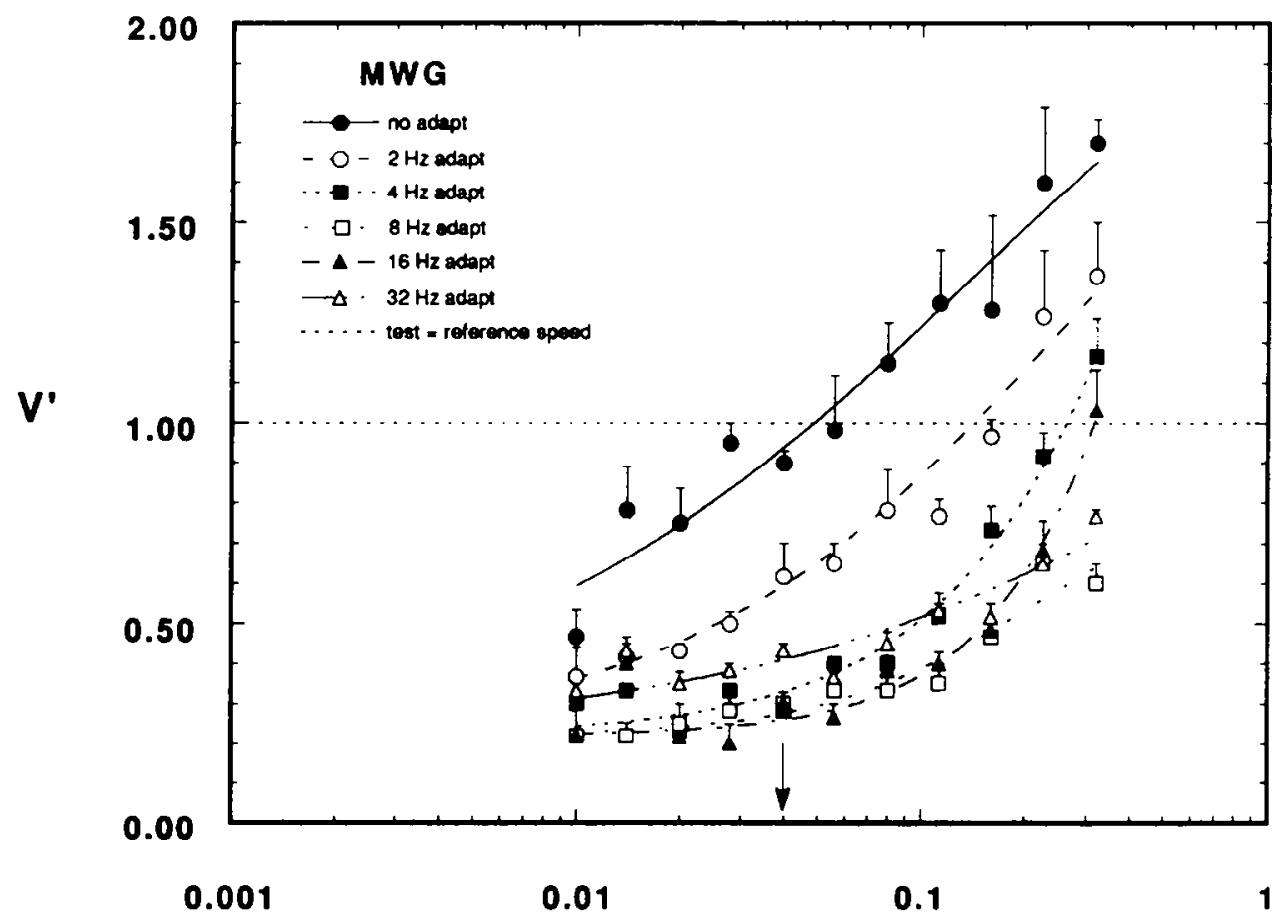

Roterence Contrast

b

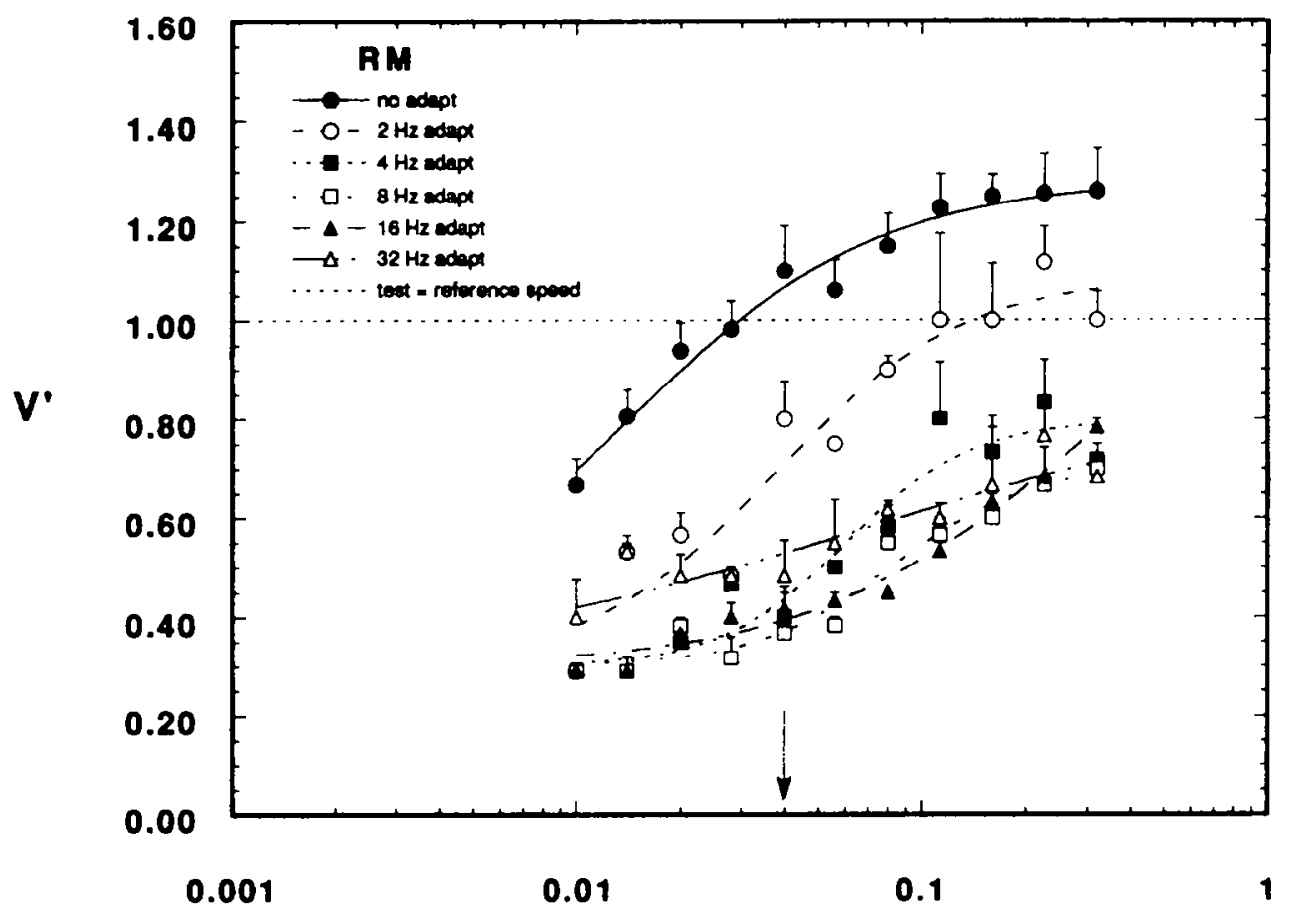

Roforence Contrast

FIGURE 7. The perceived speed $V^{\prime}$, defined in equation (4), as a function of the contrast of the reference grating for test gratings of 0.04 contrast (arrow). Solid circles show the results for the unadapted condition and the other symbols show the findings after adaptation to a high-contrast (0.4) grating drifing rightwards at various rates (see inset). The symbols show the means of three measurements and the error bars + ISE. The results for observer MWG are shown in (a) and for RM in (b). An analysis of variance for repeated measures was applied to the results to determine the statistical significance of the main effects of adaptation frequency and reference contrast on the perceived speed judgements. Both Adaptation Frequency $[F(4,4)=170.3, P=0.0001]$ and Reference Contrast $[F(10,10)=11.96, P=0.0003]$ had a highly significant effect on the speed matches. The interaction between these main effects was also highly significant $[F(40,40)=2.57, P=0.0017]$ indicating that the shapes of the $V^{\prime}$ vs contrast functions differed for the different adaptation conditions. 
TABLE 1. Parametric values $( \pm 1 \mathrm{SE})$ of the hyperbolic ratio [equation (5)] used to describe the functions relating $V$ and contrast prior to and after adaptation for the two observers tested

\begin{tabular}{|c|c|c|c|c|c|c|c|c|c|c|}
\hline \multirow{2}{*}{$\begin{array}{l}\text { Adapt } \\
\text { condition }\end{array}$} & \multirow[b]{2}{*}{$V_{\operatorname{trin}}^{*}$} & \multicolumn{2}{|r|}{ MWG } & \multirow[b]{2}{*}{$n$} & \multirow[b]{2}{*}{$r$} & \multirow[b]{2}{*}{$V_{\min }^{\prime}$} & \multicolumn{2}{|r|}{ RM } & \multirow[b]{2}{*}{$n$} & \multirow[b]{2}{*}{$r$} \\
\hline & & $V_{\max }^{\prime}$ & $C_{0.5}$ & & & & $V_{\max }^{*}$ & $\mathrm{C}_{0,5}$ & & \\
\hline No adapt & 0.22 & $2.0 \pm 0.06$ & $0.085 \pm 0.008$ & $0.68 \pm 0.07$ & 0.97 & 0.29 & $0.98 \pm 0.03$ & $0.014 \pm 0.001$ & $1.19 \pm 0.17$ & 0.98 \\
\hline $2 \mathrm{~Hz}$ & & $2.1 \pm 0.08$ & $0.27 \pm 0.02$ & $0.81 \pm 0.06$ & 0.98 & & $0.84 \pm 0.12$ & $0.039 \pm 0.012$ & $1.29 \pm 0.44$ & 0.95 \\
\hline $4 \mathrm{~Hz}$ & & $2.1 \pm 0.11$ & $0.36 \pm 0.03$ & $1.28 \pm 0.33$ & 0.98 & & $0.64 \pm 0.18$ & $0.077 \pm 0.035$ & $1.64 \pm 0.69$ & 0.95 \\
\hline $8 \mathrm{~Hz}$ & & $1.0 \pm 0.14$ & $0.46 \pm 0.13$ & $0.87 \pm 0.18$ & 0.89 & & $0.60 \pm 0.03$ & $0.147 \pm 0.015$ & $1.20 \pm 0.18$ & 0.97 \\
\hline $16 \mathrm{~Hz}$ & & $2.0 \pm 0.23$ & $0.42 \pm 0.05$ & $1.65 \pm 0.26$ & 0.95 & & $0.65 \pm 0.02$ & $0.153 \pm 0.010$ & $1.20 \pm 0.11$ & 0.98 \\
\hline $32 \mathrm{~Hz}$ & & $1.0 \pm 0.09$ & $0.45 \pm 0.13$ & $0.60 \pm 0.08$ & 0.91 & & $0.69 \pm 0.03$ & $0.120 \pm 0.017$ & $0.62 \pm 0.10$ & 0.91 \\
\hline $\begin{array}{c}\text { Mean of as } \\
\text { values }\end{array}$ & & 1.64 & 0.39 & 1.04 & 0.94 & & 0.64 & 0.107 & 1.19 & 0.95 \\
\hline $\begin{array}{l}\text { Adapted u } \\
\text { ratio }\end{array}$ & & 0.82 & 4.6 & 1.53 & & & 0.65 & 7.7 & 1.00 & \\
\hline
\end{tabular}

grating and a value above 1.0 implies that the speed of the test grating had to be increased in order to match the perceived speed of the reference grating. It should be noted that in all of the experiments reported in the following the physical speed of the reference grating remained unchanged. $V^{\prime}$ is an estimate of the perceived speed of the reference grating and variations in $V^{\prime}$ thus reflect the effects of stimulus contrast and adaptation state on this perceived speed.

In Fig. 7, solid circles present the results determined before adaptation. $V^{\prime}$ increases as the contrast of the reference grating increases. That means that the perceived speed of the reference grating with constant physical speed increases with increasing contrast. For observer MWG [Fig. 7(a)], $V^{\prime}$ approaches unity when the reference contrast is equal to that of the test contrast (indicated by the arrow pointing to the value on the abscissa). $V^{\prime}$ significantly exceeds unity when reference contrast is greater than the test contrast. Observer RM [Fig. 7(b)] exhibits a tendency for values to exceed unity already at low reference contrasts. The other symbols in Fig. 7 show the findings for the adaptation conditions with an adapting frequency from 2 to $32 \mathrm{~Hz}$ rightwards (see inset). Adaptation to an iso-directional drifting grating shifts the perceived speed vs contrast function downwards and to the right and this shift increases with increasing adaptation frequency up to $16 \mathrm{~Hz}$. For $32 \mathrm{~Hz}$ adaptation frequency, the curve reapproaches the unadapted values. Both observers show a similar dependency on contrast and adaptation state.

The curves are the best fitting hyperbolic ratios of the form:

$$
V^{\prime}=V_{\min }^{\prime}+V_{\max }^{\prime}\left[\frac{C^{n}}{\left(C^{n}+C_{0.5}^{n}\right.}\right]
$$

where $V_{\min }^{\prime}$ is the lower asymptotic value of $V^{\prime}$, $V_{\text {min }}^{\prime}+V_{\text {max }}^{\prime}$ is the upper asymptotic value of $V^{\prime}, C_{0.5}$ is the semisaturation constant and the exponent $n$ determines the steepness of the function when $V^{\prime}$ is plotted against $\log C$.

As was evident in the results shown in Fig. 2, the rightward direction threshold is some value above zero. Before adaptation, the mean of this threshold for subjects $M W G$ and RM was 0.21 and $0.63 \mathrm{~Hz}$, respectively.
Individual differences in direction thresholds are also reflected, though to a lesser extent, in $V_{\text {min }}^{\prime}$, which was found to be 0.22 for subject MWG and 0.29 for subject RM. $V_{\min }^{\prime}$ is the lower asymptotic value of $V^{\prime}$ for the different adaptation conditions. $V_{\min }^{\prime}$ reflects the value at which the observer perceived the visible reference stimulus as almost stationary. As reference contrast approached the detection threshold level, it was difficult for the subjects to detect the reference stimulus, thus making $V^{\prime}$ difficult to define. In such cases, we have substituted $V^{\prime}{ }_{\min }$ for $V^{\prime}$ (for MWG these were the values at 0.01 reference contrast following 8 and $16 \mathrm{~Hz}$ adaptation, for RM the values at 0.01 and 0.014 reference contrasts following $2-16 \mathrm{~Hz}$ adaptation and $4-16 \mathrm{~Hz}$ adaptation, respectively). These points have been excluded from the curve-fitting procedure and thus do not contribute to the values in Table 1.

Table 1 presents the values of the parameters in equation (5) for the two subjects tested. $V_{\min }^{\prime}$, as described above, was given a constant value for each subject. $V_{\text {max }}^{\prime}, C_{0.5}$ and $n$ were determined by leastsquares criterion and iteration. The goodness of fit is depicted by the correlation coefficient $r$. The ratios of the mean adapted and unadapted values are given in the last row of Table 1. Adaptation to an iso-directional drifting grating increases the semisaturation constant and to a lesser extent. the exponent of the function, whereas it reduces the maximal perceived speed.

It is clear from the findings in Fig. 7 and Table 1 that the speed of the adapting grating determines the extent to which the perceived speed vs contrast function is shifted by adaptation. We reanalysed the results shown in Fig. 7 by plotting the relative adaptation effect $R_{l}$, where

$$
R_{V^{\prime \prime}}=\frac{V_{\text {unadapted }}^{\prime}}{V_{\text {adapted }}^{\prime}}
$$

as a function of the adaptation temporal frequency. The mean $R_{v}$. values averaged over the different reference contrast levels are shown by the continuous line and solid symbols in Fig. 8 for the two observers tested. We also calculated the $R_{V}$ values for three different reference contrast levels: for low contrasts $(0.02-0.04)$, for medium contrasts $(0.056-0.113)$ and for high contrasts 
a

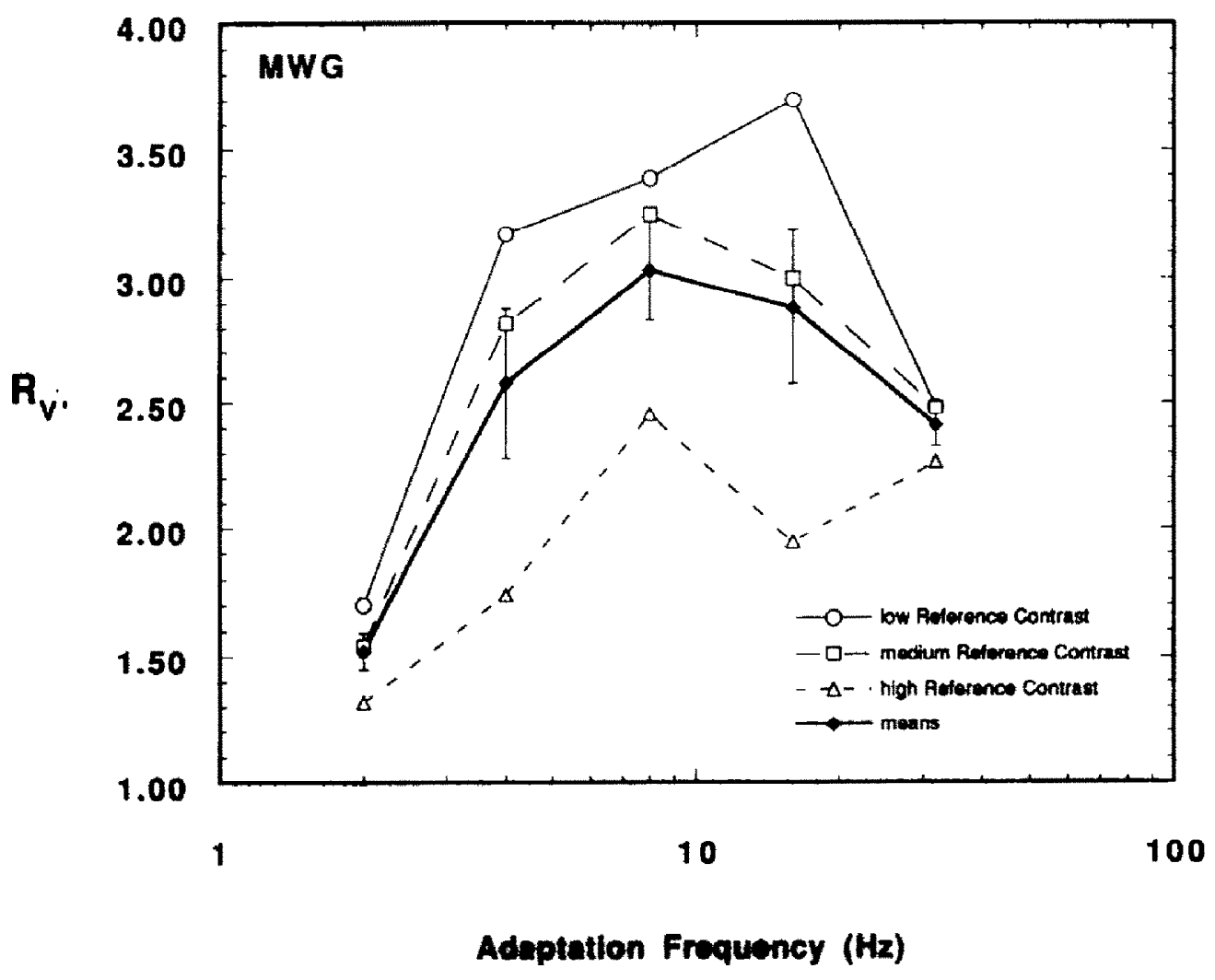

b

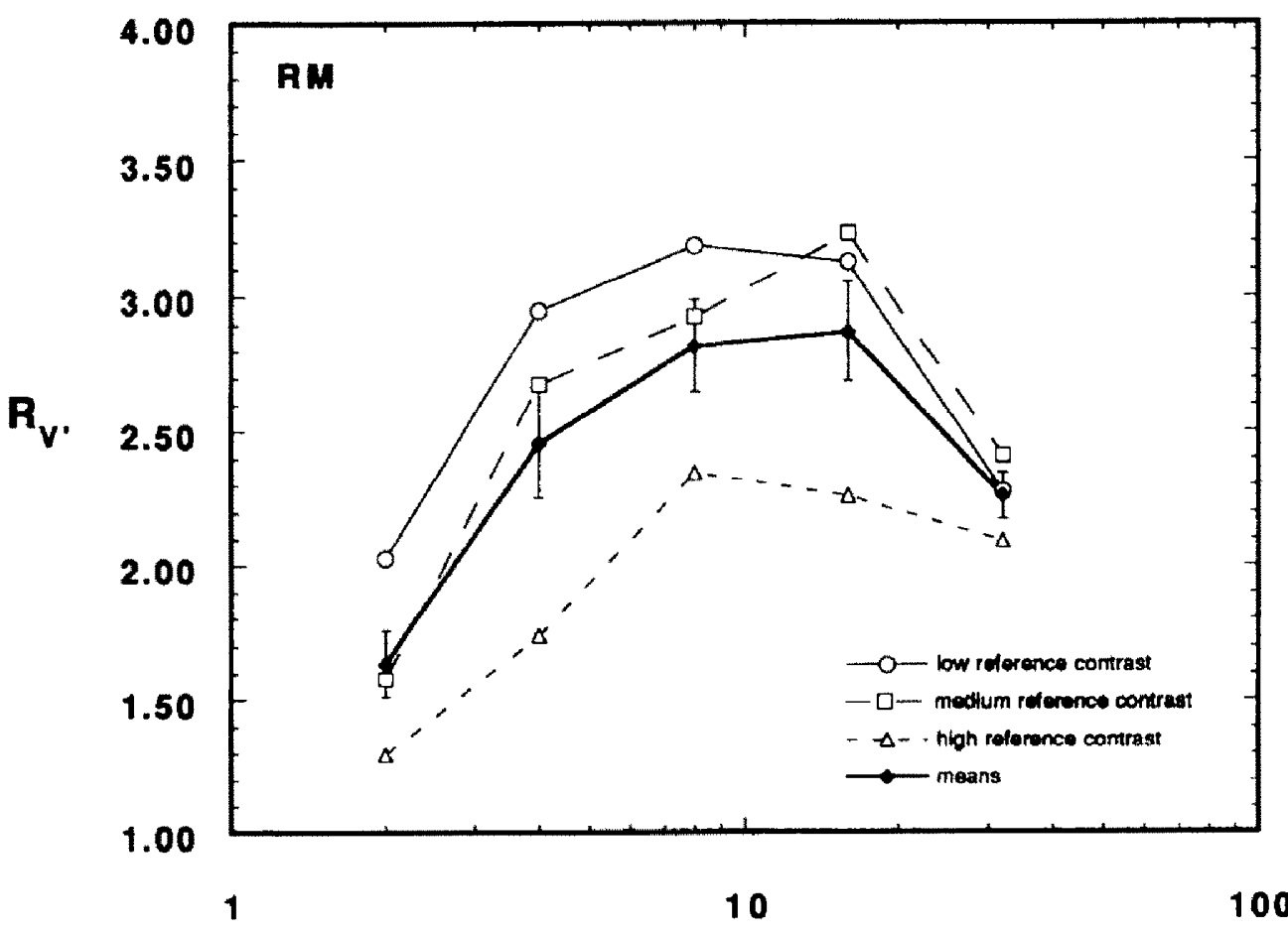

Adaptation Froquency (Hz)

FIGURE 8. The relative adaptation effect, $R_{r}$, as defined in equation (6), as a function of the adaptation temporal frequency for observer MWG (a) and RM (b). The different open symbols show the mean of nine measurements (three runs for each of three reference contrast values) for the low $(0.02,0.028,0.04)$, medium $(0.056,0.08,0.113)$ and high $(0.16,0.226,0.32)$ contrast levels separately (see inset). The solid symbols show the means over all nine reference contrast levels. 


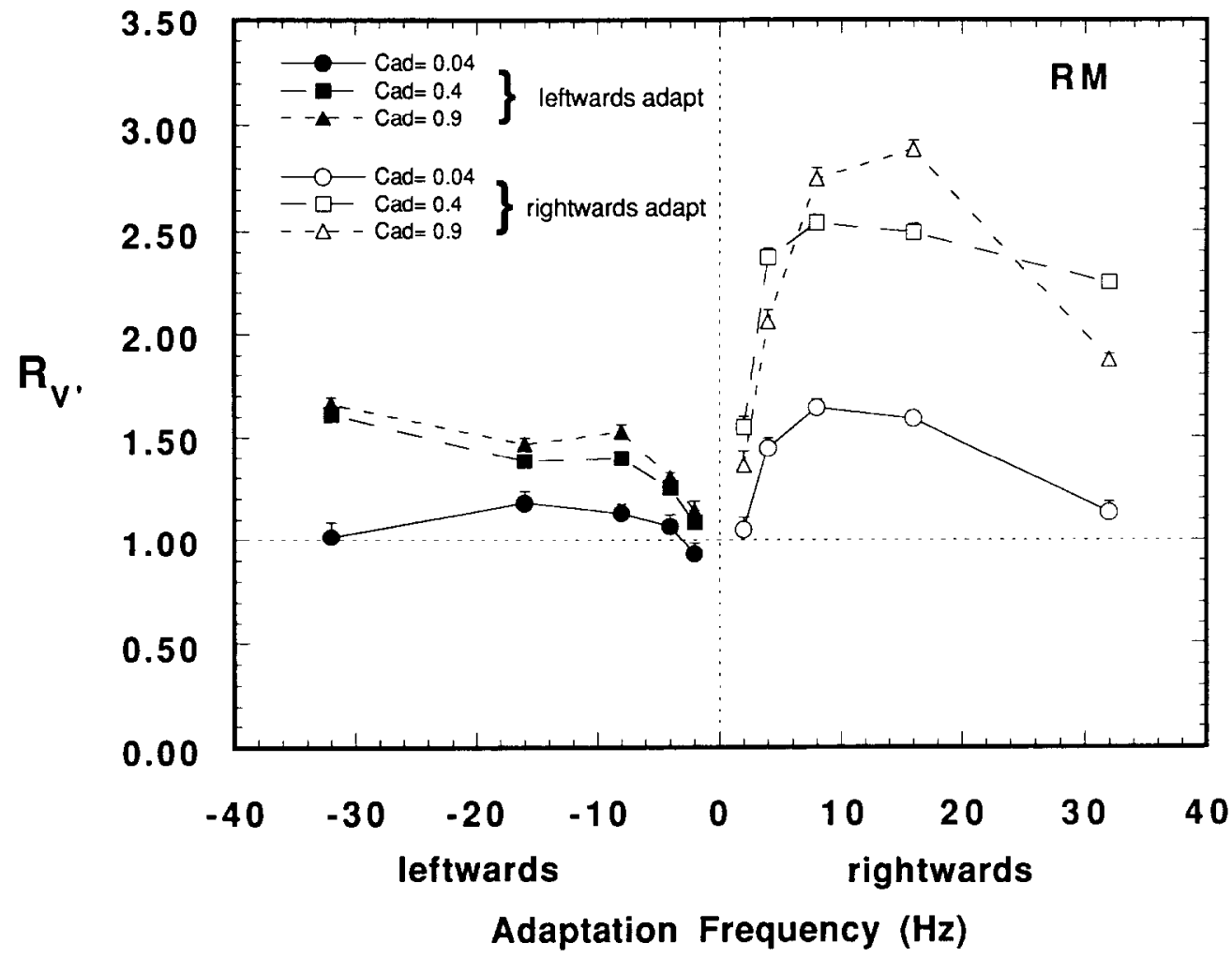

b

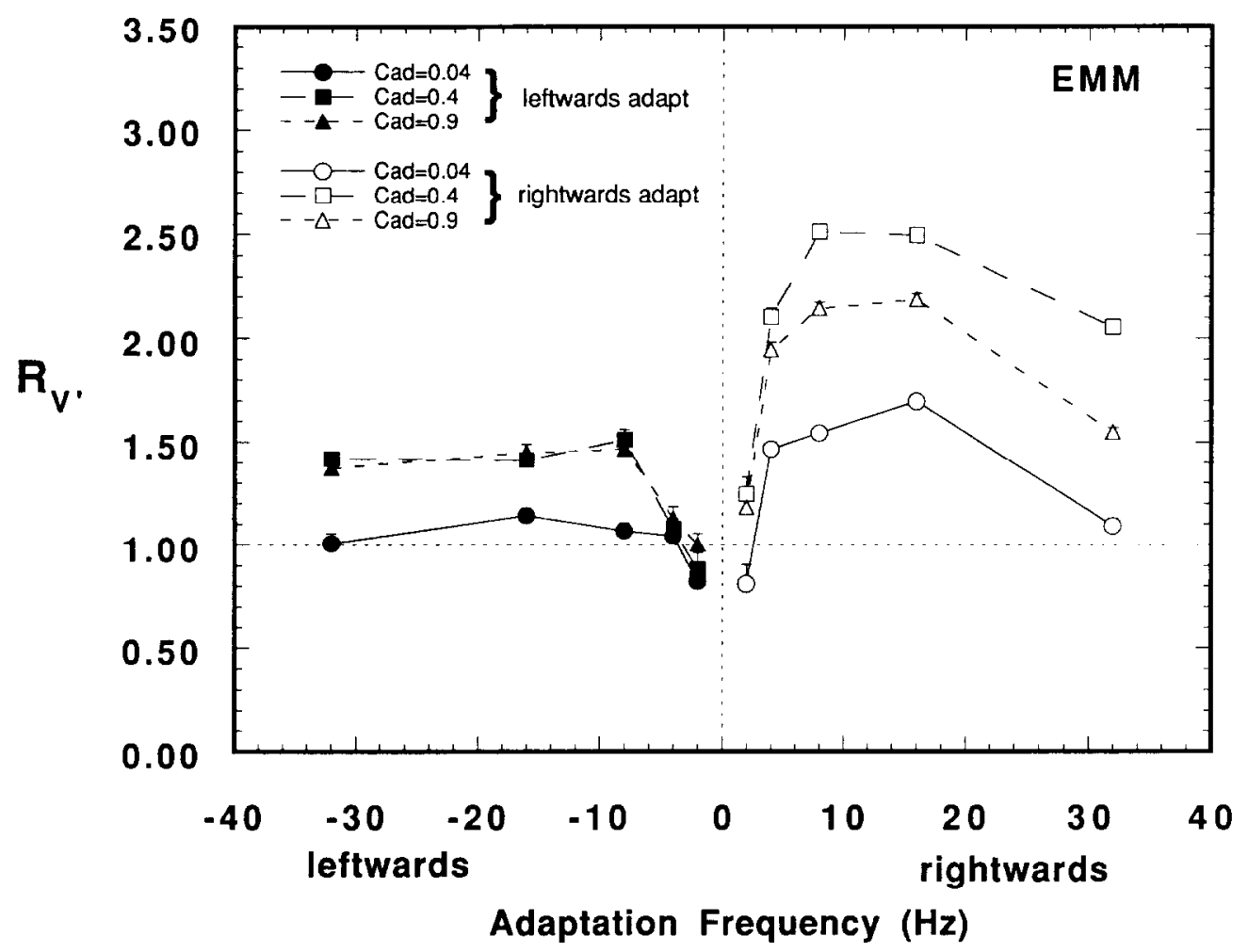

FIGURE 9. The relative adaptation effect $R_{V^{\prime \prime}}$, as defined in equation (6), as a function of the adaptation temporal frequency and direction. The different symbols designate the three adapting contrast levels used (see inset). Solid symbols show the results for the condition of leftward (contra-directional) adaptation and open symbols show the findings for rightward (iso-directional) adaptation. The symbols show the mean of 15 measurements (three runs for each of five reference contrast levels). Error bars designate +1 SE. The horizontal dotted line indicates an $R=1.0$, i.e. no adaptation effect. Results for observer RM are shown in (a) and those for EMM in (b). An analysis of variance for repeated measures (SuperANOVA. version 1.11, Abacus Concepts) was applied to the results to determine the statistical significance of the main effects of adaptation direction, adaptation contrast, adaptation frequency and reference contrast on the perceived speed judgements. The main effects Adaptation Direction $[F(1,3)=189.6, P=0.0008]$, Adaptation Contrast $[F(2,3)=8.82, P=0.05]$, Adaptation Frequency $[F(5,15)=72.7, P=0.0001]$ and Reference Contrast $[F(4,12)=61.1, P=0.0001]$ had a highly significant effect on the speed matches. The interactions between the main effects Adaptation Direction and Adaptation Frequency $[F(5,15)=30.0, P=0.0001]$ and between Adaptation Frequency and Reference Contrast $[F(20.60)=3.8$. 
$(0.16-0.32)$. The results of this analysis are shown by the dashed curves and open symbols in Fig. 8 (see inset). The results indicate that the effect of adaptation increases with adaptation frequency up to 8-16 Hz, after which it begins to decline. Both subjects exhibit a clear bandpass tuning of the adaptation effect. The adaptation effect is greatest for low reference contrasts and declines for medium to high contrasts. The overall shape of the temporal frequency tuning curves and the position of the maxima, are similar for the different reference contrast levels. The results of an analysis of variance for repeated measures support these conclusions. The main effects of adaptation frequency $[F(4,4)=28.2, P=0.0034]$ and reference contrast $[F(8,8)=6.34, P=0.0085]$ are higly significant. There is a significant interaction between these terms $[F(32,32)=2.05, P=0.023]$, suggesting that the shape of the functions varies somewhat with reference contrast level.

\section{Experiment III: effects of adaptation direction and adap-} tation contrast on perceived speed

The effects of adaptation to a drifting grating have been shown to be selective to the direction and contrast of the adapting grating, as already evident in the early studies (Breitmeyer, 1973; Sekuler \& Ganz, 1963). We compared the effects of adaptation to rightward and leftward drifting gratings with $0.04,0.4$ and 0.9 contrast levels. Figure 9 presents the results of these measurements for two observers; for observer RM in Fig. 9(a) and observer EMM in Fig. 9(b). The relative adaptation effect $R_{V}$ [as defined in equation (6)] is shown as a function of the drift rate of the adapting grating. Negative values on the abscissa indicate leftward (contra-directional) adaptation direction and positive values designate rightward (isodirectional) drift. The reference and test gratings always drifted to the right. The test contrast was, in this experiment, 0.08 and the reference contrast varied from 0.02 to 0.32 , each step increasing by a factor of 2 . There is a clear dependency of the relative adaptation effect on adaptation direction: adaptation in the same direction reduces the perceived speed of drifting gratings more than adaptation in the opposite direction. The adapting contrast also contributes to the effects of adaptation on perceived speed. Interestingly, fairly robust effects on the perceived speed were already evident for the low contrast adapting grating of 0.04. A similar tuning of the adaptation effect as that evident in Fig. 8 was found at the three different adapting contrasts (note that the abscissa is linear in Fig. 9). The frequency selectivity of adaptation is not as pronounced for contra-directional adaptation (see below).

The results of an analysis of variance are summarized in the legend of Fig. 9. The interaction between the main effects adaptation direction and adaptation frequency indicates that the shape of the temporal frequency tuning curves of the adaptation effect depends on the relative direction of adaptation, whereas the interaction between adaptation frequency and reference contrast suggests that the shapes of the $V^{\prime}$ vs contrast functions differed for the different adaptation frequencies. This latter result was described above for iso-directional adaptation (Fig. 7). a

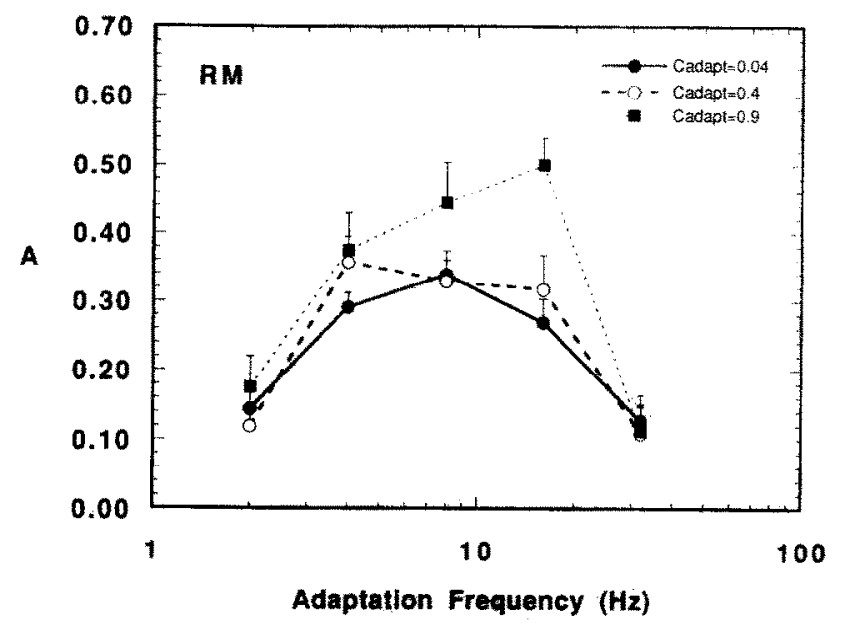

b

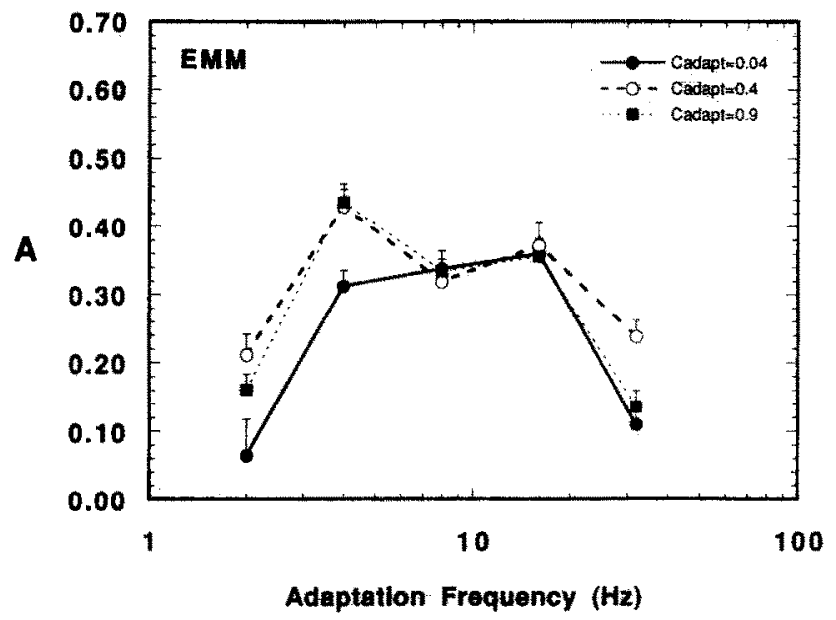

FIGURE 10. The asymmetry index $A$, defined in equation (7), as a function of adaptation temporal frequency for observer RM (a) and observer EMM (b). The different symbols designate the three adapting contrast levels used (see inset). Each symbol indicates the mean of 15 measurements, obtained from three runs for iso- and three runs for contra-directional adaptation, with the five reference contrast levels

$0.02,0.04,0.08,0.16$ and 0.32 . Error bars represent + ISE.

To provide a quantitative description of the relative change in perceived velocity following iso-directional vs contra-directional adaptation, we define the asymmetry index:

$$
A=1-\left[\frac{V_{\text {isodirectional }}^{\prime}}{V_{\text {conira-directional }}^{\prime}}\right]
$$

If the perceived velocity is affected to an equal extent by iso- and contra-directional adaptation then $A \approx 0$. whereas a substantial reduction in $V^{\prime}$ for isodirectional adaptation without a concomitant change for contradirectional adaptation would lead to an $A \approx 1$. Figure 10 portrays $A$ as a function of the adaptation drift rate, which is based on results taken from Fig. 9. The results indicate that the extent of directional asymmetry of the effects of adaptation on perceived velocity depends on the adaptation frequency. The asymmetry index $A$ is reduced for adaptation frequencies of 2 and $32 \mathrm{~Hz}$ and greatest for adaptation frequencies between 4 and $16 \mathrm{~Hz}$. This observation was supported by statistical analysis (ANOVA for repeated measures), which revealed a 
highly significant effect of adaptation frequency on the asymmetry index $A[F(4,12)=36.3, P=0.0001]$. This result confirms that the effects of adaptation to a drifting grating have their most pronounced directional selectivity for drift rates centred around $8 \mathrm{~Hz}$. The magnitude and shape of these functions are not significantly affected by the adaptation contrast [main effect of adaptation contrast, $F(2,3)=2.4, \quad P>0.2$; interaction between adaptation contrast and adaptation frequency, $F(8,12)=0.9, P>0.5]$.

Figure 11 refers to these same results, but now the asymmetry index $A$ is plotted as a function of the reference contrast level. Although there is a tendency for $A$ to increase with decreasing reference contrast level, this effect is only marginally significant $[F(4,12)=3.1$, $P=0.055]$. The lack of a significant interaction with adaptation frequency $[F(16,48)=1.6, P>0.1]$ and adaptation contrast $[F(8,12)=0.49, P>0.8]$ suggests that

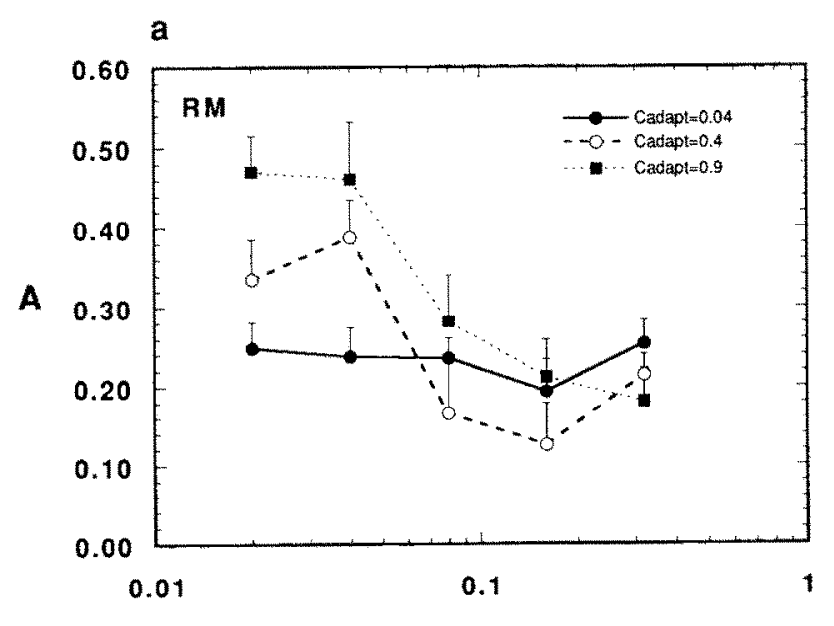

Reference Contrast

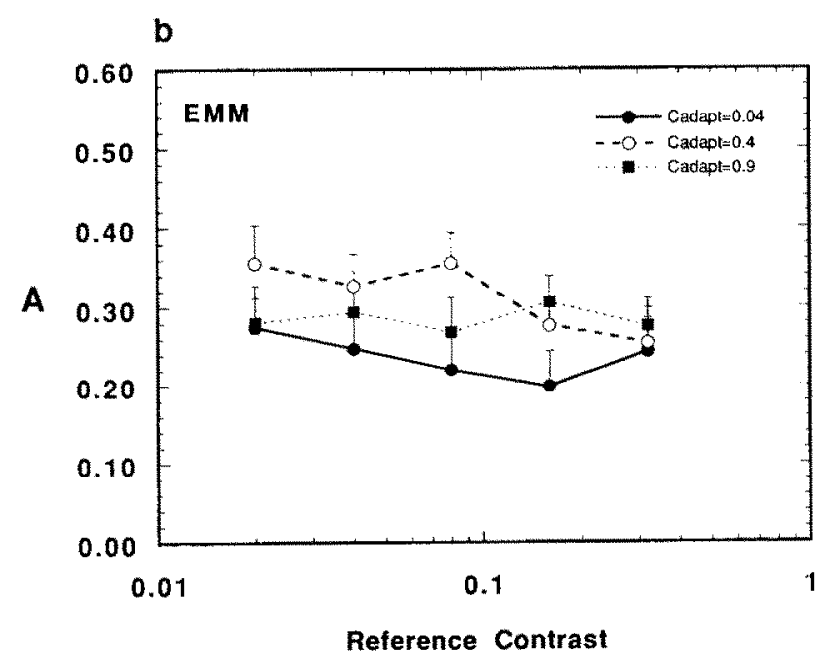

FIGURE 11. The asymmetry index $A$, defined in equation (7), as a function of reference contrast for observer RM (a) and observer EMM (b). The different symbols designate the three adapting contrast levels used (see inset). Each symbol represents the mean of 15 measurements, obtained from three runs for iso- and three runs for contra-directional adaptation with the five adaptation temporal frequencies $2,4,8,16$ and $32 \mathrm{~Hz}$. Error bars show + 1SE. the role of reference contrast on the asymmetry of the adaptation effect is minimal.

\section{DISCUSSION}

We have explored the effects of contrast and adaptation state on three aspects of motion perception: (1) the direction threshold; (2) the speed discrimination threshold; and (3) the perceived speed of drifting gratings. We discuss these results in the following and describe how changes in the contrast transducer function can lead to these effects.

\section{Effect of contrast and adaptation on direction and speed discrimination}

Thresholds for direction and speed discrimination are for the most part independent of contrast before adaptation. Only for the lowest contrasts, those near detection threshold level, do direction and speed discrimination thresholds begin to increase. This contrast independence of discrimination performance for contrasts more than a few times higher than detection threshold has been described earlier for both stationary and moving patterns (Bowne, 1990; Gouled Smith \& Thomas, 1989). Both of these earlier studies noted that spatial- and/or temporal-frequency discrimination behave differently than contrast discrimination as the contrast of the grating stimuli to be discriminated increases. For contrasts ranging from 0.01 to 0.32 , we found that the LTM (Wright \& Gurney, 1992) is independent of contrast before adaptation (Fig. 3). In a similar manner, the speed discrimination threshold for briefly presented sine-wave gratings is also, to a considerable extent, independent of the contrast level prior to adaptation (Fig. 5). These findings suggest that the underlying neural mechanisms saturate in their response already at low contrast. Following adaptation to a drifting grating of 0.4 contrast, LTM is significantly elevated. This elevation is most pronounced for low contrast gratings (Fig. 3). Above test contrasts of 0.1 , adaptation has only a modest effect on the LTM.

We computed hyperbolic ratios for the inverse LTM and found, in the three subjects tested, that they provide a good description of the dependency of thresholds on contrast, especially for LTMs measured after adaptation. Adaptation shifts the LTM vs log contrast function to the right towards higher contrast levels. In agreement with the findings of Greenlee and Thomas (1992), who explored the effects of pattern adaptation on the relationship between spatial frequency discrimination threshold and contrast, we find that adaptation primarily acts to rescale effective contrast for performance on the direction discrimination task.

Since we measured the velocity which elicited a reliable impression of both rightward and leftward motion before and after rightward adaptation, we could compute the effect of rightward adaptation on the PSS. Under the present conditions and for the three observers tested, prior to adaptation PSS is independent of contrast and slightly positive (Fig. 4). The more positive 
value for observer RM might be due to residual adaptation, since circumstances required RM to complete his measurements in an overall shorter amount of time. For all three subjects, following rightward adaptation, PSS is shifted towards larger values of rightward drift and this adaptation effect is most pronounced for low test contrasts. This shift in the perceptual null point is another way of looking at the motion aftereffect: physically stationary gratings appear to drift leftwards after rightward adaptation and a specific value of rightward velocity will appear to the adapted observer as stationary. In agreement with these findings, it has been demonstrated earlier that the initial perceived speed of the motion aftereffect is greater at low test contrasts (Keck, Palella \& Pantle, 1976).

Adaptation to a rightward drifting grating increases the Weber fraction for speed discrimination and this increase is most pronounced for low reference contrast levels (Fig. 5). The effect of adaptation on speed discrimination thresholds is also dependent on the speed of the adapting grating. Discrimination thresholds were most greatly elevated for adaptation drift rates between 8 and $16 \mathrm{~Hz}$ (Fig. 6).

\section{Effect of contrast and adaptation on perceived speed}

In Expt III, we explored the effects of contrast level and adaptation on the perceived speed of drifting gratings. The results indicate that the perceived speed of a parafoveally viewed grating depends on its contrast level. Our findings are in close agreement with those of Thompson (1982) and Stone and Thompson (1992). We have demonstrated that the hyperbolic ratio, as expressed in equation (5), provides an adequate description of the dependency of perceived speed on contrast. Although we do not want to assert that this is the only nonlinear function that would fit our data, it has proven useful in describing the effects of adaptation on contrastdependent visual performance. Following adaptation, the function describing the relationship between the perceived speed of a grating and contrast is altered, and, as is evident in Fig. 7 and the results in Table 1, this alteration is characterized by a reduction in the maximal perceived velocity $\left(V_{\text {min }}^{\prime}+V^{\prime}{ }_{\text {inax }}\right)$, as well as an increase in the semisaturation contrast $\left(C_{0,5}\right)$. Adaptation has no effect on the exponent $(n)$ for observer RM and only a marginal effect for observer MWG. The relative adaptation effect $R_{v}$. was found to depend on the adapting temporal frequency (Fig. 8). Interestingly, the maximal adaptation effect was not found at the reference temporal frequency of $2 \mathrm{~Hz}$, but rather between 8 and $16 \mathrm{~Hz}$. The results of earlier studies (Thompson, 1983; Watson \& Robson, 1981) suggest that velocity for each direction is coded by two channels. The results of visual evoked potential recordings (VEP) before and after adaptation on drifting gratings support this idea (Müller, Göpfert \& Hartwig, 1985). In masking experiments, the detection threshold for a low temporal frequency test stimulus, which had a spatial frequency similar to that used in the present study, was maximally elevated by $7-8 \mathrm{~Hz}$ temporal modulation (Anderson \& Burr. 1985). More re- cently, Hess and Snowden (1992) and Snowden and Hess (1992) obtained maximal threshold elevations between 8 and $10 \mathrm{~Hz}$ with a variant of the previously used noise masking paradigm. These findings and the present ones suggest that the neural mechanisms providing the relevant information for the perceptual decisions at low drift rates have their maximal sensitivity around $8 \mathrm{~Hz}$.

The present findings also indicate that the effect of adaptation on the perceived speed of drifting gratings is dependent on the contrast level (Fig. 8). The adaptation effect increases as contrast decreases from high, over medium, to low levels. This result is in line with the findings presented in Fig. 4, where we showed that. following adaptation to a rightward drifting grating, the PSS takes on larger values of rightward speed as stimulus contrast decreases. After adaptation, PSS approaches the reference speed of $2 \mathrm{~Hz}$, especially for low stimulus contrast levels and as a consequence, the perceived speed is greatly reduced. This, in turn. leads to a larger $R_{1}$ value. It is interesting to note that the shape of the functions describing the relationship between $R_{1}$ and the adaptation temporal frequency is more or less invariant with reference contrast level (Fig. 8).

The findings further indicate that the effect of adaptation on the perceived velocity of drifting gratings depends on the relative direction of the reference and adaptation gratings, as well as on the contrast of the adapting grating (Fig. 9). This fact was already evident since the early studies (Breitmeyer. 1973: Sekuler \& Ganz, 1963). Iso-directional adaptation has a more pronounced effect on the perceived speed of drifting gratings than does contra-directional adaptation. The reduction in the perceived speed following contradirectional adaptation was. at first glance, unexpected. Following adaptation to a rightward drifting grating, the PSS is shifted in this direction and this can be as much as $2 \mathrm{~Hz}$ (Fig. 4). It might be assumed that a leftward drifting grating with a drift frequency of. say $1 \mathrm{~Hz}$, should be perceived as faster following rightward adaptation, since the distance to the PSS is increased. This. however, is not the case. Contra-directional adaptation generally leads to an overall reduction in the perceived velocity of subsequently viewed gratings $\left(R_{l}>1.0\right.$, see Fig. 9).

As is evident in Fig. 9, the effect of adaptation to a drifting grating has more than a single component: the resultant change in perceived velocity has (1) a contrast component; (2) a speed component; and (3) a direction component.

(1) Iso- and contra-directional adaptation always leads to a reduction in the perceived contrast of the reference grating. As we have seen in Fig. 7. a reduction in reference contrast leads to a corresponding reduction in the perceived velocity. The results in Fig. 9 further indicate that an increase in the adaptation contrast evokes a greater reduction in perceived speed. Georgeson (1985) has shown that an increase in the adaptation contrast leads to a greater reduction in perceived contrast. Therefore, we can conclude that the 
contrast component of adaptation should lead to a reduction in perceived speed.

(2) The perceived speed of a $2 \mathrm{~Hz}$ rightward drifting grating is most greatly reduced by $8-16 \mathrm{H} /$ adaptation (Fig. 9). The range of drift rates over which adaptation is most effective is, to a considerable extent, independent of the relative direction of adaptation. Here we can conclude that the speed component of motion adaptation acts to reduce the perceived speed of subsequently viewed drifting gratings.

(3) The direction-selective component to motion adaptation (corresponding to an $A>0$ ) is evident in the findings of Figs 10 and 11. Iso-directional adaptation leads to a greater reduction in perceived velocity than does contra-directional adaptation. If the direction component of motion adaptation could be isolated from the contrast and speed components, then it follows that iso-directional adaptation should lead to a reduction in perceived speed $\left(R_{l^{\prime}}>1\right)$, whereas contra-directional adaptation should induce an elevation in perceived speed. For the stimulus conditions of low adaptation contrast (0.04) and low adaptation temporal frequency $(2 \mathrm{~Hz})$, there is a suggestion in the data that contradirectional adaptation can lead to a slight increase in the perceived velocity of subsequently viewed test gratings (solid circles, Fig. 9). This aspect remains to be confirmed by further investigation.

Electrophysiological recordings of human scalp potentials evoked by the onset of a moving stimulus (motion-onset VEP) indicate that the amplitude of a negative component with a latency around $200 \mathrm{msec}$ (N200) is reduced following iso-directional adaptation (Müller, Göpfert \& Hartwig, 1986). The reduction in the amplitude of this component was more pronounced as adaptation frequency increased from 0.25 to $10 \mathrm{~Hz}$. In these experiments, the reference and adapting contrast levels were always the same. Variations in contrast between 0.05 and 0.8 had little influence on the effect of adaptation with respect to the amplitude of the N200 component. The present results indicate that the adaptation index $R_{l}$ " [equation (6)] increases with increasing adaptation contrast (Fig. 9), but decreases with increasing reference contrast (Fig. 8). A simultaneous increase in both adaptation and reference contrast would thus act to cancel their respective effects, which would leave $R_{L}$. more or less unaltered, thus corresponding to the VEP findings reported in the Müller et al. (1986) study. The amplitude of the N200 component was also found to depend on the reference contrast level (Müller \& Göpfert, 1988). The VEP-amplitude increased proportionally to the logarithm of contrast, but reached a saturation level already at 0.05 contrast. Contrary to these VEP findings, the psychophysical results shown in Fig. 7 and those of Stone and Thompson (1992) indicate that the perceived speed of drifting gratings continues to increase for high contrasts.

It is of interest in this context to note that $50 \%$ of the cells in the macaque extrastriate area MT, a region known to be involved with motion processing, have a semisaturation constant less than 0.07 and a median exponent of 3.0. These values are compared to 0.33 and 2.4 , respectively, for the same parameters of the contrast-response functions of cells in striate visual cortex (Sclar, Maunsell \& Lennie, 1990). Such differences would imply that cells in extrastriate visual areas in the primate occipito-temporal cortex should saturate, on average, at lower contrast levels than those in striate cortex. The low semisaturation constants of MT cells might prevent contrast-dependent variations in activity in these direction-selective motion detectors. Adaptation to a drifting grating might shift the contrast-response function of these cells, thereby increasing the range over which their response depends on contrast. In comparison, the results of the perceived speed estimates yield semisaturation constants before adaptation that vary from 0.014 (observer RM) to 0.085 (observer MWG) and increase by a factor ranging from $7.6(\mathrm{RM})$ to 4.6 (MWG) after adaptation.

Also of interest here are studies that have compared the directional selectivity of single cells in the areas $\mathrm{V} 1$, V2 and MT. The argumentation, made above with respect to the three components of motion adaptation, suggests that the contrast- and speed-dependent components of adaptation should be related to the bidirectional cell responses, whereas the direction component of motion adaptation should be related to the uni-directional cell responses. In the physiological literature, the direction index (DI) is defined as:

$$
D I=1 \quad\left[\begin{array}{c}
R_{\text {anti-precerred }} \\
R_{\text {preclerred }}
\end{array}\right] .
$$

where $R$ is the neural response. DI is thus comparable to our asymmetry index [equation (7)].

$\mathrm{A} D \mathrm{DI} \approx 1$ would mean that a cell responds only in the preferred direction, whereas DI $\approx 0$ implies that a cell responds with the same magnitude in both preferred and anti-preferred direction. Single-unit recordings in a large number of cells in area MT of the macaque indicate that these cells are directionally selective (Albright, 1984; Britten, Shadlen, Newsome \& Movshon, 1992; Maunsell \& van Esscn, 1983; Mikami, Newsome \& Wurtz, 1986). These authors calculated the DI to describe the directional selectivity of the examined cell population and found an average index of 1.0 , which indicates a strong uni-directional response to stimulus motion. These results suggest that exposure to stimulus motion in the "anti-preferred" direction should evoke no overall response and therefore cause no adaptation, in MT cells with a maximal sensitivity in the "preferred" direction. Albright (1984) compared the directional selectivity of cells in area V1 and MT in the macaque and found a mean DI of only 0.55 in area V1, compared to 1.0 for MT neurons. Foster, Gaska, Nagler and Pollen (1985) found that the DI for cells in area V2 falls in between these two values. For the present investigation, this result suggests that the contrast- and speed-dependent components of motion adaptation could be accounted for by the response properties of $\mathrm{V} 1$ and $\mathrm{V} 2$ cells, whereas the direction component of motion adaptation is more likely related to the response characteristics of 


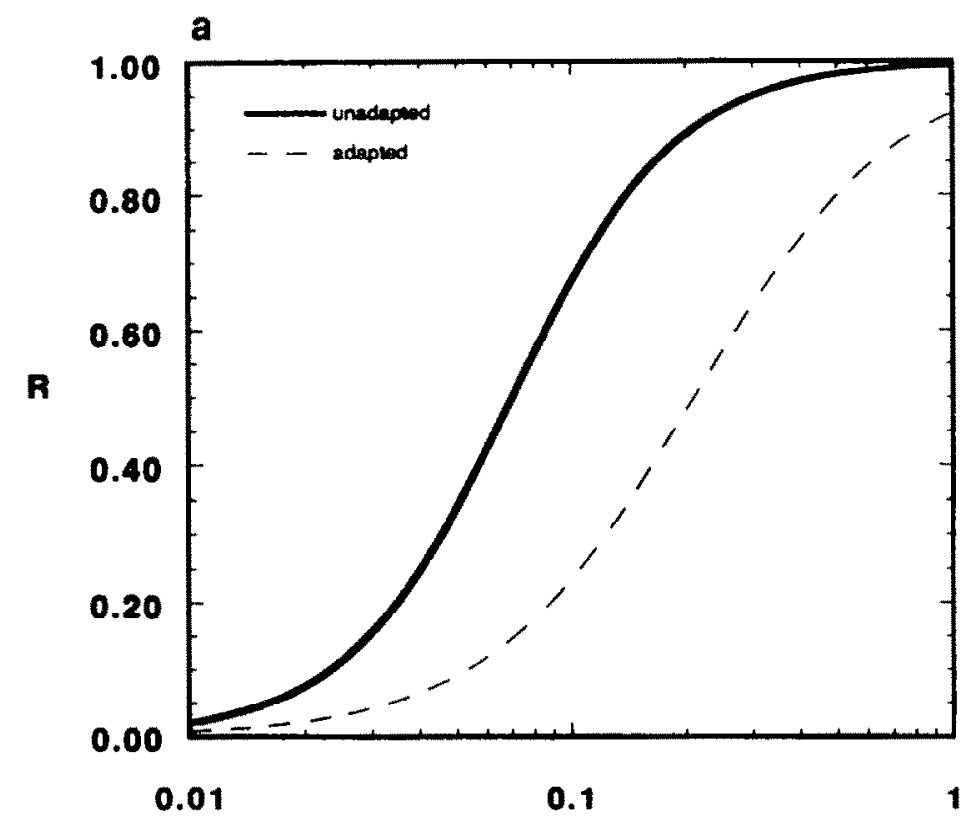

\section{Contrast}

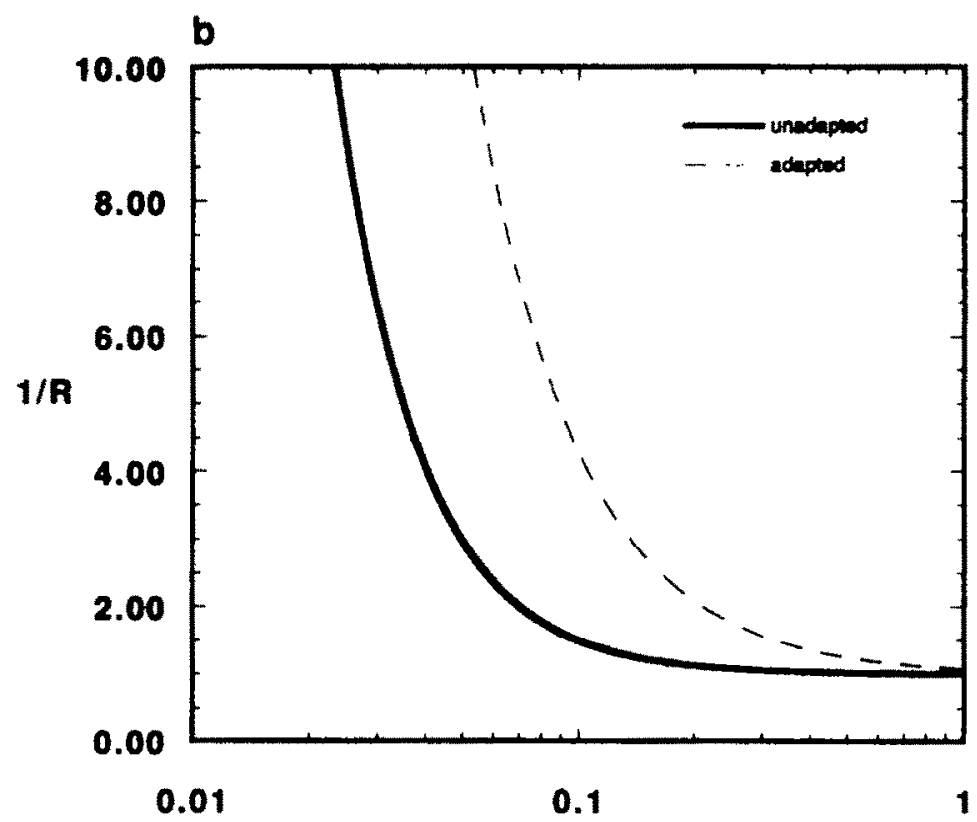

FIGURE 12. Schematic illustrations of the effects of adaptation on the underlying contrast transducer functions and the consequences of the shift in the transducer function on relationship between $\log$ contrast and $R$ the neural response (a) and $1 / R(b)$.

area MT cells. Note, however, that the large differences between observers exclude the possibility of making any firm conclusions in this respect.

In Expts I and II, we have shown that adaptation to moving stimuli can have a modifying effect on direction and speed discrimination for low contrasts. The results of Expt III indicate that prior adaptation has an overall attenuating effect on the perceived speed of moving stimuli. Our findings seem to suggest that velocity is encoded by an intensity dependent mechanism, high response intensity designating high speed and low response intensities indicating low speed. Following adap- tation to a drifting grating the response amplitude for a given contrast level is reduced. Although this response reduction can, in special cases, improve the ability to detect the direction of briefly presented moving highcontrast gratings (Derrington \& Goddard, 1989), it leads to an overall nonveridical perception of absolute speed.

Although we do not attempt in this investigation to provide a quantitative model of the effects of adaptation on the different aspects of motion perception studied here, Fig. 12 serves to illustrate in a qualitative manner our current ideas. Figure 12(a) schematically portrays putative neural responses to periodic stimuli as a func- 
tion of the log contrast level. These are hyperbolic ratios described in its general form as:

$$
R=R_{\max }\left[\frac{C^{n}}{\left(C^{n}+C_{0.5}^{n}\right.}\right]
$$

The "unadapted" and "adapted" functions differ by a factor of 3 in their semisaturation constants, the adapted curve taking on a larger value. The effect this shift in the transducer function has on the ratio $R$ (a) and the inverse of $R$ (b) are shown in Fig. 12. At first approximation, such a shift in the underlying transducer functions mimics the effect adaptation has on motion perception. Again, we would like to emphasize that there is only a qualitative similarity between the shifting transducer function and our results. The physiological mechanism underlying this gain control still needs to be described, but recent models of adaptation have emphasized the role of response pooling over mechanisms tuned to different orientations (Heeger, 1992; Wilson \& Humanski, 1993).

In summary we have explored the effects of contrast and adaptation to drifting gratings on three aspects of motion perception: direction thresholds, speed discrimination and perceived speed. Adaptation to a drifting grating increases the LTM and shifts the PSS towards higher velocities of motion in the adapted direction. Adaptation to a drifting grating increases the speed discrimination threshold for test contrasts below 0.1 , having a maximal effect for adaptation drift rates between 8 and $16 \mathrm{~Hz}$. The hyperbolic ratio provides an adequate description of the dependency of the LTM and the Weber fractions for speed discrimination on contrast. We have shown that adaptation shifts the contrast-response functions towards higher contrast levels. Such a shift implies a multiplicative gain control which reduces the input signal by a constant factor and as a consequence reduces the maximal perceived velocity. This shift in the operating range not only accounts for the illusory reduction in perceived velocity, it can also account for the elevation in direction and velocity discrimination thresholds at low contrasts we have described.

\section{REFERENCES}

Albrecht, D. G.. Fartar, S. B. \& Hamilton. D. B. (1984). Spatial contrast adaptation characteristics of neurons recorded in the cat's visual cortex. Journal of Physiology, 347, 713-739.

Albright. T. D. (1984). Direction and orientation selectivity of neurons in visual area MT of the macaque. Journal of Neurophysiology, 52. 11061130

Anderson, S. J. \& Burr, D. C. (1985). Spatial and temporal selectivity of the human motion detection system. Vision Research, 25. 11471154.

Barlow. H. B. \& Hill, R. M. (1963). Evidence for a physiological explanation of the waterfall phenomenon and figural aftereffects. Nature, 200, 1345-1347.

Blakemore. C. \& Campbell, F. W. (1969). On the existence of neurons in the human visual system selectively sensitive to the orientation and size of retinal images. Journal of Physiology, London. 203, 237.260.

Boulton, J. C. \& Baker, C. L. Jr (1991). Motion detection is dependent on spatial frequency not size. Vision Research, 31, 77-87.
Bowne, S. F. (1990). Contrast discrimination cannot explain spatial frequency, orientation or temporal frequency discrimination. Vision Research, 30, 449-461.

Breitmeyer, B. G. (1973). A relationship between the detection of size, rate, orientation and direction in the human visual system. Vision Research, 13, 41-58.

Britten, K. H., Shadlen, M. N., Newsome, W. T. \& Movshon, J. A. (1992). The analysis of visual motion: A comparison of neuronal and psychophysical performance. Journal of Neuroscience, 12. 4745-4765.

Cavanagh. P. \& Favreau, O. E. (1985). Color and luminance share a common motion pathway. Vision Research, 25. 1595-1601.

Cavanagh, P., Tyler, C. W. \& Favreau, O. E. (1984). Perceived velocity of moving chromatic gratings. Journal of the Optical Society of America A, 1,893 899 .

Derrington. A. M. \& Goddard, P. A. (1989). Failure of motion discrimination at high contrasts: Evidence for saturation. liwion Research. 29.1767-1776.

Diener, H. C. Wist, E. R., Dichgans, J. \& Brandt, T. (1976). The spatial frequency effect on perceived velocity. Vision Research, it, $169-176$.

Ferrera, V. P. \& Wilson, H. R. (1991). Perceived speed of moving two-dimensional patterns. Vision Research, 31, 877893.

Foster, K. H., Gaska, J. P., Nagler, M. \& Pollen, D. A. (1985). Spatial and temporal frequency selectivity of neurons in visual cortical areas $\mathrm{V} 1$ and $\mathrm{V} 2$ of the macaque monkey. Jotmal of Physiology, London. $365,331-363$

Georgeson. M. A. (1985). The effect of spatial adaptation on perceived contrast. Spatial Vision, I, 103112.

Gouled Smith, B.\& Thomas, J. P. (1989). Why are some spatial discriminations independent of contrast? Joumat of the Optical Society of America A, 6. 713-724.

Greenlee, M. W. (1992). Spatial frequency discrimination of bandlimited periodic targets: Effects of stimulus contrast, bandwidth and retinal eccentricity. Vision Research, 32, 275283.

Greenlee. M. W. \& Ileitger. F. (1988). The functional role of contrast adaptation. Vision Rescarch, 28,791 797.

Greenlec. M. W. \& Thomas, J. P. (1992). Effect of pattern adaptation on the spatial frequency discrimination. Sommat of the Optical Society of America A, 9,857 862 .

Heeger, D. I. (1992). Normalization of cell responses in cat striate cortex. Visual Neuroscience, 9. 181197.

Hess, R. F. \& Snowden, R. J. (1992). Temporal properties of human visual filters: Number, shapes and spatial covariation. I'isiom Research, 32, 47-59.

Howard, E. (1989). Perception of sinusoidal gratings as a function of Gaussian truncation. Unpublished, University of California, Los Angeles, Calif.

Hunzelmann. N. \& Spillmann, L. (1984). Movement adaptation in the peripheral retina. Vision Research, 24, 17651769

Johnston. A. \& Wright. M. J. (1985). Lower thresholds of motion for gratings as a function of eccentricity and contrast. Vision Research, $25.179-185$.

Johnston, A. \& Wright, M. J. (1986). Matching velocity in central and peripheral vision. Vision Research, 26, 1099-1109.

Keck, M. J., Palella, T. D. \& Pantle, A. (1976). Motion aftereffect as a function of the contrast of sinusoidal gratings. Virion Resesert, 16, 187-191.

Lieberman. H. \& Pentland. A. P. (1982). Microcomputer-based estimation of psychophysical thresholds: The best PEST. Behatior Research Methods, Instruments, and Computers, 14. 21-25.

Marcelja, S. (1980). Mathematical description of the responses of simple cortical cells. Journal of the Optical Society of America, 70, 12971300

Mauisell, J. H. R. \& van Essen, D. C. (1983), Functional properties of ncurons in the middle temporal visual area of the macaque: 1 . Selectivity for stimulus direction. speed and orientation. Journal of Neurophysiology, 49, 11271147.

Mikami, A.. Newsome, W., T. \& Wurtz, R. H. (1986). Motion selectivity in macaque visual cortex. I. Mechanisms of direction and speed selectivity in extrastriate area MT. Journal of Neurophysiology, 55. $1308-1327$. 
Müller, R. \& Göpfert, E. (1988). The influence of grating contrast on the human cortical potential visually evoked by motion. Acta Neurobiologiae Experimentalis, 48, 239-249.

Müller, R., Göpfert, E. \& Hartwig, M. (1985), VEP-Untersuchungen zur Kodierung der Geschwindigkeit bewegter Streifenmuster im Kortex des Menschen. Zeitschrift für EEG \& EMG, 16, 75-80.

Müller, R., Göpfert, E. \& Hartwig, M. (1986). The effect of movement adaptation on human cortical potentials evoked by pattern movement. Acta Neurobiologiae Experimentalis, 46, 293-301.

Ohzawa, I., Sclar, G. \& Freeman, R. D. (1985). Contrast gain control in the cat's visual system. Journal of Neurophysiology, 54, 651-667.

Pantle, A. \& Sekuler, R. (1968). Size detecting mechanisms in human vision. Science, 162, 1146-1148.

Sclar, G., Lennie, P. \& DePriest, D. D. (1989). Contrast adaptation in the striate cortex of macaque. Vision Research, 29, 747-755.

Sclar, G., Maunsell, J. H. R. \& Lennie, P. (1990). Coding of image contrast in central visual pathways of the macaque monkey. Vision Research, 30, $1-10$

Sekuler, R. \& Ganz, L. (1963). Aftereffect of seen motion with a stabilized retinal image. Science, 139, 419-420.

Sekuler, R. \& Pantle, A. (1967). A model for after-effect of seen movement. Vision Research, 7, 427-439.

Smith, A. T. \& Edgar, G. K. (1990). The influence of spatial frequency on perceived temporal frequency and perceived speed. Vision Research, 30, 1467-1474.

Snowden, R. J. \& Hess, R. F. (1992). Temporal frequency filters in the human peripheral visual field. Vision Research, 32, 61-72.

Stone, L. S. \& Thompson, P. (1992). Human speed perception is contrast dependent. Vision Research, 32, 1535-1549.

Thomas, J. P. (1983). Underlying psychometric functions for detecting gratings and identifying spatial frequency. Journal of the Optical Society of America, 73, 751-758.
Thompson, P. (1981), Velocity after-effects: The effects of adaptation to moving stimuli on the perception of subsequently seen moving stimuli. Vision Research, 21, 337-345

Thompson, P. (1982). Perceived rate of movement depends on contrast. Vision Research, 22, 377-380.

Thompson, P. (1983). Discrimination of moving gratings at and above detection threshold. Vision Resecirch, 23, 15.33 15.38.

Troscianko, T. \& Fahle, M. (1988). Why do isoluminant stimuli appear slower? Journal of the Optical Society of America A, $5,871880$.

Tynan, P. D. \& Sekuler, R. (1982). Motion processing in peripheral vision: Reaction time and perceived velocity. Fivim Reseurch. 22, $61-68$.

Watson, A. B. \& Robson, J. G. (1981). Discriminatson at threshold: Labelled detectors in human vision. Vision Research, 21.1115. 1122.

Wilson, H. R. \& Humanski, R. (1993), Spatial frequency adaptation and contrast gain control. Vision Research, 33, 11331149.

Wright, M. J. \& Gurney, K. N. (1992), Lower threshold of motion for one and two dimensional patterns in central and peripheral vision. Vision Research, 32, 121-134

Acknowledgements-This research was supported by the Deutsche Forschungsgemeinschaft, SFB 325, B4. M.W.G. is a Feodor-Lynen fellow of the Alexander von Humboldt Foundation. R.M. was funded during his stay in Freiburg by a special travel grant from the Deutsche Forschungsgemeinschaft and the Stifterverband für die Deutsche Wissenschaft to encourage collaboration between laboratories in the West and East parts of Germany. The authors would like to thank Dr Lothar Spillmann for his help and advice, as well as Hans-Jorg Lang and Eric Munro for participating as observers 